\title{
Influence of post-synthetic modifications on the composition, acidity and textural properties of ZSM-22 zeolite
}

Pablo del Campo ${ }^{\mathrm{a}}$, Pablo Beato ${ }^{\mathrm{b} *}$, Fernando Rey $^{\mathrm{c}}$, Maria Teresa Navarro $^{\mathrm{c}}$, Unni Olsbye ${ }^{\mathrm{a}}$, Karl Petter Lillerud ${ }^{\mathrm{a}}$ and Stian Svelle $\mathrm{a}^{* *}$

${ }^{a}$ SMN Centre for Materials Science and Nanotechnology, Department of Chemistry, University of Oslo, N-0315 Oslo, Norway

${ }^{b}$ Haldor Topsфe AS, Nymфllevej 55, DK-2800 Kgs. Lyngby, Denmark

${ }^{c}$ Instituto de Tecnología Química, Universitat Politècnica de València, Camino de Vera s.n., 46022 Valencia, Spain

* Corresponding author. Tel.: +45 452720 00; fax: +45 45272999

** Corresponding author. Tel.: +4722855454; fax: +47228554 41

E-mail addresses: pabb@topsoe.dk (P. Beato), stian.svelle@kjemi.uio.no (S. Svelle)

\section{Abstract}

In this work, an extensive investigation of the preparation of a large body of desilicated ZSM-22 zeolites and their basic characterization is presented. We investigate the effects of the properties of the starting zeolite, and we employ mixtures of $\mathrm{NaOH}$ with $\mathrm{CTAB}$ or TBAOH as well as subsequent acid washings to create mesoporous zeolites. Scanning and transmission microscopy and nitrogen adsorption revealed that the crystal morphology of the starting zeolite appears to be the dominant parameter which influences the mesopore generation. Mesopores were effectively created within the rodlike commercial crystallites, whereas the thinner dimensions of the needle-shaped particles of the in house prepared zeolite represent an obstacle for an intra-mesopore creation. The alkaline, surfactant-assisted or combined NaOH/TBAOH desilication methods resulted in mesopores with different shape and size from the commercial 
zeolite. The sequential acid washing generally resulted in increased the micropore volume with respect to the desilicated samples. Elemental analysis showed that extraframework Al species were generated upon the desilication treatments, which are eventually removed by the acid treatment. The acidity studied by FTIR demonstrated that this occurs without a marked modification of the Brønsted acidity, whereas the concentration of surface silanol hydroxyl groups is increased. The comparison between the total $\mathrm{Al}$ concentration and the amount of $\mathrm{Al}$ in acidic sites shows that the acidity was recovered after the acid washing and suggests that original non-acidic Al species in the starting materials may have a role in the formation of both Lewis and extra-framework species upon desilication.

\section{Keywords}

Zeolite ZSM-22

Mesoporous

Desilication

Post-synthetic

Crystal morphology

\section{Introduction}

Zeolites are crystalline aluminosilicates composed of linked $\mathrm{SiO}_{4}$ and $\mathrm{AlO}_{4}$ tetrahedras. The connections between these building blocks result in three-dimensional frameworks with a well-defined arrangement of pores or channels of molecular dimensions. It is possible to introduce catalytically active sites within these pores, such as Brønsted acidity [1-3]. The combination of the porous and the acidic properties makes zeolites 
highly useful in different applications, such as separation, ion exchange and catalysis [2-4]. It is the presence of differently sized micropores that give rise to the use of zeolites as shape selective catalyst [5], but the micropore system also limits the applicability of zeolites as catalysts, often imposing diffusion constraints and preventing access to the active sites of the zeolite crystal [4]. In order to overcome this limitation, the introduction of a secondary network of mesopores interconnected with the existing micropores is desirable. This can be achieved by a variety of synthetic and post synthesis approaches. The preparation of such hierarchical zeolites and their improved catalytic performance have been extensively reviewed [6-19]. Desilication with $\mathrm{NaOH}$ is the most employed technique to create mesoporosity in zeolites, owing to the efficiency and simplicity of this method. Most research focusses on the 3-dimensional MFI zeolite [20-39], but studies involving other 3-dimensional (BEA [28, 40], FAU [41, 42]), 2 dimensional (FER [28, 43]) and 1-dimensional (EUO [44], IFR [45], MOR [28, 46, 47], MTW [48]) zeolites have been reported.

The TON topology is characterized by a pore structure of non-intersecting 5.7 x $4.6 \AA$ 10-member ring channels and crystallizes preferentially as needles, with the micropore channels running in the direction of the needles [49-53]. Zeolites having the TON topology (ZSM-22, NU-10, Theta-1) are certainly strongly affected by diffusion limitations. Therefore mesopore development is highly attractive to improve their catalytic performance. However, only a few studies have been reported for mesoporous TON zeolites [54-62]. Verboekend et al. [58] demonstrated that, by using adequate conditions, desilication with $\mathrm{NaOH}$ yields ZSM-22 zeolites with increased mesopore surface, attributed to the presence of both intra- and inter-crystalline mesopores. Matias et al. [56] and Sá Couto et al. [57] also showed that desilication of the NU-10 zeolite results in the creation of additional porosity. However, the mesoporosity development 
was limited compared to other topologies. The low desilication efficiency (defined as the external surface introduced per percent of weight loss) was attributed both to the particular needle-like morphology of the ZSM-22 $[19,47,58]$ and to uneven Al distribution. The re-deposition of the initially extracted framework Al in the zeolite crystal as extra framework aluminum species (EFAl) during desilication caused a slight reduction in Brønsted acidity and an increase in Lewis acid sites in all cases. Recently, Dyballa et al. performed desilication of ZSM-22 using both $\mathrm{NaOH}$ and $\mathrm{KOH}$ and found significant reductions of the density of acid sites [61] Verboekend et al. [58] observed that these extra-framework species blocked most of the micropore volume of ZSM-22. However, a subsequent acid treatment with $\mathrm{HCl}$ successfully restored the pristine microporosity by removing the amorphous species. The acid washing of desilicated zeolites has also been studied for other topologies with similar beneficial effects on the porosity and, consequently, on the catalytic behavior [37, 44, 63-65].

The $\mathrm{Si} / \mathrm{Al}$ represents a key parameter for successful mesopore generation. For MFI zeolites, an optimal $\mathrm{Si} / \mathrm{Al}$ ratio for efficient mesopore creation was found to be $25-50$ $[14,24,26,29]$. Lower $\mathrm{Si} / \mathrm{Al}$ results in excessive protection against the attack of the hydroxide anion on the partly negatively charged $\mathrm{Si}-\mathrm{O}-\mathrm{Al}$ bonds, whereas highly siliceous zeolites are prone to excessive dissolution. However, for zeolites with a high concentration of intergrowth defects, the effects of the $\mathrm{Si} / \mathrm{Al}$ ratio were minor and mesopores were formed preferentially along defects boundaries [31, 35, 38].

Desilication with $\mathrm{NaOH}$ is known also to occasionally result in the destruction of the zeolite framework and the treatment is thus not specific with respect to the size of the mesopores developed. Instead, treatments combining $\mathrm{NaOH}$ with other agents, such as surfactants or hydroxides of various bulky organic (tetraalkylammonium) cations, tend to result in controlled mesopore size. For example, by adding a cetyltrimethylamonium 
bromide $(\mathrm{CTAB})$ surfactant to the alkaline solution, the mesopore size can be tuned to a range of pore size distributions [66-68]. A recrystallization process, where the zeolite is partially dissolved in $\mathrm{NaOH}$ solution and then treated with $\mathrm{CTAB}$ leads to mesopores of controlled size [69-71]. For highly siliceous ZSM-5 zeolites, tetraalkylammonium (tetrabutylammonium and tetrapropylammonium, TBA and TPA) cations can play a dominant role as pore direction agent (PDA) resulting in mesopores of small diameter and narrow size distribution [72-79]. Recently, Liu et al. reported mesopore formation for ZSM-22 by extending the dissolution and recrystallization process with CTAB to the TON topology [54].

In this work, we present an extensive investigation of the preparation of desilicated ZSM-22 zeolites and their basic characterization. We investigate the effects of the properties of the starting zeolite, and we employ mixtures of $\mathrm{NaOH}$ with $\mathrm{CTAB}$ or $\mathrm{TBAOH}$ as well as subsequent acid washings, thereby encompassing the entire array of desilication methods reported previously. By creating a large body of samples, we attempt to look for general observations and to eliminate the effects of outliers caused by the occasional erratic outcome of various post synthesis treatments. The samples are characterized using scanning and transmission microscopy, elemental analysis, nitrogen adsorption and IR spectroscopy with different probe molecules. In an accompanying article, we discuss the performance of these materials as catalysts for the conversion of methanol to hydrocarbons [80-82] and attempt to correlate catalytic performance to characterization data. The benefits of mesoporosity for TON zeolites have been demonstrated for several catalytic processes, such as isomerization, cracking reactions, and MTO [54-57, 59, 61]. This literature is more extensively presented in the introduction to the accompanying article. 


\section{Experimental Section}

\subsection{Parent ZSM-22 zeolite}

Commercially available and in-house made ZSM-22 zeolites are the two starting materials for this work, and will be referred as $c$-ZSM-22 and $m$-ZSM-22, respectively. $c$-ZSM-22 with a $\mathrm{Si} / \mathrm{Al}$ ratio of 49 was supplied by Zeolyst International. $m$-ZSM-22 was synthesized following the procedure described elsewhere [82, 83]. $1.05 \mathrm{~g}$ of aluminium sulfate (J. T. Baker) was added to $10.9 \mathrm{~g}$ of distilled water under vigorous stirring. An aqueous solution of $2.4 \mathrm{~g}$ of potassium hydroxide (Sigma-Aldrich) dissolved in $10.89 \mathrm{~g}$ distilled water was prepared and mixed with the previous solution. Another solution of $6.26 \mathrm{~g}$ of diaminooctane (DAO from Fluka) dissolved in $43.6 \mathrm{~g}$ of distilled water was prepared and added to the first mixture. To the resulting solution, $28.5 \mathrm{~g}$ of Ludox AS-30 colloidal silica (Sigma Aldrich) diluted with $16.16 \mathrm{~g}$ of distilled water was added under vigorous stirring. This final mixture was stirred for $30 \mathrm{~min}$. After $24 \mathrm{~h}$ ageing at room temperature, the resulting gel, with a composition of $8.9 \mathrm{~K}_{2} \mathrm{O}$ : $1.0 \mathrm{Al}_{2} \mathrm{O}_{3}: 90 \mathrm{SiO}_{2}: 3 \mathrm{~K}_{2} \mathrm{SO}_{4}: 27.3 \mathrm{DAO}: 3588 \mathrm{H}_{2} \mathrm{O}$, was transferred to $40 \mathrm{ml}$ Teflon-lined stainless steel autoclaves. Crystallization was carried out at $160{ }^{\circ} \mathrm{C}$ for $72 \mathrm{~h}$ in a tumbling oven $(37 \mathrm{rpm})$. Teflon coated magnets were placed inside the autoclaves to enhance the gel mixing. The product was recovered by filtration, extensively washed with deionized water and dried overnight at $90{ }^{\circ} \mathrm{C}$. The materials were calcined under a flow of pure oxygen at $550{ }^{\circ} \mathrm{C}$ for $12 \mathrm{~h}$ to remove the structure directing agent. The temperature of the oven was increased stepwise, in $100{ }^{\circ} \mathrm{C}$ segments up to $400{ }^{\circ} \mathrm{C}$ and $50{ }^{\circ} \mathrm{C}$ segments above $400{ }^{\circ} \mathrm{C}$, with a hold time of 30 min between each temperature increase. The received commercial material was calcined following the same procedure. 
The acidic form of the zeolites was produced by three consecutive ion exchanges with a $1 \mathrm{M} \mathrm{NH}_{4} \mathrm{NO}_{3}$ solution at $75^{\circ} \mathrm{C}$ for $2 \mathrm{~h}$ and calcined again in static air at $550{ }^{\circ} \mathrm{C}$ for $2 \mathrm{~h}$.

\subsection{Post-synthesis treatments}

\subsubsection{Desilication with different treatments}

Desilication of the calcined commercial and as-synthesized ZSM-22 materials was carried out using three different approaches. For clarification, sample codes and desilication conditions are summarized in Table 1. First, the parent material was treated with $0.2 \mathrm{M}$ or $0.5 \mathrm{M} \mathrm{NaOH}$ aqueous solutions at $80{ }^{\circ} \mathrm{C}$ during $2 \mathrm{~h}$, with a solid to liquid ratio of $33 \mathrm{~g} \mathrm{l}^{-1}$ (-at coded series). For the second approach, the desilication was carried out in a mixture of $0.05 \mathrm{M} \mathrm{CTAB}$ and $0.25 \mathrm{M}$ or $0.5 \mathrm{M} \mathrm{NaOH}$ at $80{ }^{\circ} \mathrm{C}$ for $24 \mathrm{~h}$, and 50 $\mathrm{g}^{-1}$ (-ats coded series). In the third approach, the parent samples were treated with a mixture of $0.08 \mathrm{M} \mathrm{TBAOH}$ and $0.12 \mathrm{M} \mathrm{NaOH}$ at either $65^{\circ} \mathrm{C}$ or $80{ }^{\circ} \mathrm{C}$ during $0.5 \mathrm{~h}$, and $30 \mathrm{~g} \mathrm{l}^{-1}$ (-tba coded series). The conditions were selected according to literature [56, 58] and preliminary experimental work. After the treatments, the suspension was quenched in ice bath, washed with deionized water until neutral $\mathrm{pH}$, filtered and dried overnight at $60{ }^{\circ} \mathrm{C}$. The organic-assisted treated samples were then calcined to remove the surfactant template under a flow of pure oxygen at $550{ }^{\circ} \mathrm{C}$ for $12 \mathrm{~h}$ using a heating rate of $1.8{ }^{\circ} \mathrm{C} / \mathrm{min}$ to reach $550{ }^{\circ} \mathrm{C}$.

\subsubsection{Sequential acid washing}

A fraction of each desilicated sample was submitted to acid washing in $0.1 \mathrm{M}$ aqueous $\mathrm{HCl}$ solution at $65{ }^{\circ} \mathrm{C}$ for $6 \mathrm{~h}$, with $10 \mathrm{~g} \mathrm{l}^{-1}$. The resulting product was washed with distilled water until neutral $\mathrm{pH}$ and dried overnight at $70{ }^{\circ} \mathrm{C}$. For comparative purposes, 
the parent zeolite was also treated in $\mathrm{HCl}$ using the same conditions. The samples submitted to the sequential acid treatment are coded with the suffix $-\mathrm{HCl}$. The desilicated and acid washed samples were brought to the protonic form as described in Section 2.1. As an example of the sample notation, samples prepared from commercial ZSM-22, with the lower CTAB concentration and with or without acid washing are denoted as $c$-ZSM-22-ats 1-HCl or $c$-ZSM-22-ats1, respectively.

\subsection{Characterization methods}

The crystallinity of the materials was investigated by Powder X-ray diffraction (PXRD). The measurements were performed using a Siemens Bruker D5000 instrument in a Bragg-Brentano geometry and $\mathrm{Cu} \mathrm{K}_{\alpha 1}$ radiation $(\lambda=1.5406 \AA)$. The $\mathrm{Si} / \mathrm{Al}$ ratio was determined by elemental analysis using an Agilent Technologies 4100 microwave plasma-atomic emission spectrometer (MP-AES). The morphology of the zeolite crystals was analyzed by scanning electron microscopy (SEM) and transmission electron microscopy (TEM). SEM micrographs were recorded on a SU8230 FE-SEM instrument working at $5-10 \mathrm{kV}$, using beam deceleration mode. The samples were prepared by placing a small amount of powder on a carbon tape on SEM stubs. TEM images were recorded using a CM300 FEG-TEM operating at $300 \mathrm{kV}$ or in a Philips CM-10 microscope operating at $100 \mathrm{kV}$. A small amount of the sample was crushed in a mortar and dispersed in ethanol, which was ultrasonicated for 20 seconds to further break up the agglomerates. A few drops of this solution were placed on a TEM Cu-grid coated with lacey carbon placed on a piece of filter paper. The ethanol was allowed to evaporate for 10 minutes before the TEM grid was stored in ambient conditions. The BET surface area and pore volume were determined from $\mathrm{N}_{2}$ physisorption measurements at $77 \mathrm{~K}$ in a range of relative pressures of $0-0.99$ on a Belsorp-mini II 
instrument. The BJH method was used to determine the mesopore size distribution and the t-plot method allowed determining the micro- and mesoporous volumes and the specific surface area. Before each measurement, the samples were outgassed under vacuum at $80{ }^{\circ} \mathrm{C}$ for $1 \mathrm{~h}$ and at $150{ }^{\circ} \mathrm{C}$ for $4 \mathrm{~h}$. The nature, accessibility, and concentration of acid sites were investigated using Fourier transformed infra-red (FTIR) spectroscopy. FTIR measurements were performed on a Bruker Vertex 80 instrument with MCT detector. The powder samples in the proton form were pressed into selfsupporting wafers and placed in an in-house designed quartz cell with $\mathrm{KBr}$ window. Samples were activated in-situ at $150{ }^{\circ} \mathrm{C}$ for $1 \mathrm{~h}$, at $300{ }^{\circ} \mathrm{C}$ for $1 \mathrm{~h}$ and at $450{ }^{\circ} \mathrm{C}$ for $1 \mathrm{~h}$ under vacuum. For the qualitative studies, $\mathrm{CO}$ was adsorbed on the sample at liquid nitrogen temperature. After dosing, $\mathrm{CO}$ was gradually pumped out of the cell while spectra were recorded at regular intervals. The spectra were normalized to the overtone modes at wavenumber $1750-2000 \mathrm{~cm}^{-1}$. Quantification was performed by adsorption of pyridine at room temperature, followed desorption and evacuation in-situ at $200{ }^{\circ} \mathrm{C}$ for $2 \mathrm{~h}$ under vacuum, to remove the physisorbed species. All spectra were corrected for water and $\mathrm{CO}_{2}$ present in the sample compartment and normalized to the weight of the wafers.

\section{Results and discussion}

\subsection{Parent material description}

The properties of the starting material represent a key variable in the present study. An extensive characterization of the two parent ZSM-22 zeolites is given in this initial section.

The commercial (c-ZSM-22) and in house prepared ( $m$-ZSM-22) zeolites have Si/Al ratios of 49 and 38, respectively, which are within the optimal $\mathrm{Si} / \mathrm{Al}$ ratio found for 
mesopore generation for MFI zeolites [19, 24, 29]. Both samples consist of a highly crystalline ZSM-22 structure [50-53], as indicated by the diffractograms shown in Fig. S1. The peak seen around 21.5 degrees for both samples is ascribed to cristobalite impurity which commonly co-exists with ZSM-22 [50, 58, 83].

The crystal morphology of the two zeolites was investigated by SEM. The commercial sample shows a complex morphology, where rod-like particles of dimensions of about 2-4 $\times 0.5 \mu \mathrm{m}$ appear to be an agglomeration of more defined nanorods with lengths from $600 \mathrm{~nm}$ and less than $500 \mathrm{~nm}$ in width (Fig. S2a,b). These nanorods can be interpreted as the primary particles which are afterwards aligned into the rod-like bigger crystals. This mode of crystallization of the ZSM-22 zeolite was previously described by Hayasaka et al. [84]. The in house prepared zeolite displays more defined needleshaped crystals of about 100-500 nm in length and less than $100 \mathrm{~nm}$ in thickness (Fig. S3a). TEM micrographs confirm the different morphology for the two parent zeolites. The agglomeration of crystals with different thickness up to $200 \mathrm{~nm}$ is evidenced for $c$ ZSM-22 (Fig. 1a), whereas $m$-ZSM-22 is composed of defined nanosized crystals with a broad distribution of lengths but with regular thickness of about 30-60 nm (Fig. 1 e).

Both parent samples exhibit a type-I nitrogen isotherm (Fig. 2 left panels) characteristic of a microporous material [85]. The $m$-ZSM-22 isotherm displays a low uptake at $p / p_{0}<0.1$ and a steep increase in uptake at $p / p_{0}>0.9$. The former is indicative of the limited microporosity of this sample and the latter suggest the presence of intercrystalline mesopores, which should be ascribed to the space between the aggregated nanocrystals. It should be noted that the zig-zag shape observed in the large pore region of the BJH plots for the in house prepared sample (Fig. 3 bottom panels) do not represent true mesoporosity but is considered an artifact of the analysis. Indeed, the BJH pore size distribution is the first derivative of the accumulative volume. Then, a very 
small jump in the isotherm could give a sharp increase in the $\mathrm{BJH}$, but without any significance. The BJH plot also shows limited and broad mesopores around $2 \mathrm{~nm}$ for the commercial sample (Fig. 3 top). Table 2 and Fig. 4 shows that the total surface area of 232 and $163 \mathrm{~m}^{2} \mathrm{~g}^{-1}$ of the commercial and in house prepared zeolite, respectively, are in the expected range for zeolitic materials and that he external surface area contributes to about $15 \%$ of the total surface area for both samples. Noteworthy, the inset on Fig. $2 \mathrm{a}$ shows a defined hysteresis loop for the $c$-ZSM-22 sample. Generally, the hysteresis is associated with the creation of mesopores [86-88]. However, $c$-ZSM-22 is in fact purely microporous. Hysteresis with closure point at $p / p_{0} \sim 0.42$ is often misinterpreted, in particular when only the adsorption branch of the isotherm is applied. Real mesopores should lead to reversibility for both the adsorption and desorption branches [87]. In this case, the sharp peak at $2 \mathrm{~nm}$ observed in the BJH mesopore size distribution calculated with the desorption branch (Fig. S6 top panels) is not present when using the adsorption branch (Fig. 3 top panels). The hysteresis observed may be related to the phase transition from a disordered "fluid" to a more ordered $\mathrm{N}_{2}$ phase, and does not indicate real mesoporosity.

The FT-IR spectra of the $c$-ZSM-22 and $m$-ZSM-22 zeolites activated at $450{ }^{\circ} \mathrm{C}$ are shown in Fig. 5. Two well-differentiated features can be distinguished in the $\mathrm{OH}$ stretching region for both samples: an asymmetric band at $3700-3745 \mathrm{~cm}^{-1}$ and a broader band centered at $3600 \mathrm{~cm}^{-1}$. This corresponds to the mode of isolated silanol groups and to bridging $\mathrm{Al}(\mathrm{OH}) \mathrm{Si}$ groups, giving rise to strong Brønsted acid sites (BAS), respectively [89-91]. The band associated with the isolated silanols exhibits a tail on the low frequency side, indicating the existence of silanol groups in different environments in the zeolite framework. Previous spectroscopic studies using pyridine and the bulky 2,4,6-collidine as probe molecules for determining the location of the 
silanol groups in ZSM-5, concluded that the absorption band at $3745 \mathrm{~cm}^{-1}$ can be assigned to isolated silanols located on the external zeolite surface, whereas lower frequency bands at $3700-3735 \mathrm{~cm}^{-1}$ are associated with silanol groups located inside the micropores, which might be present as internal framework defects [31, 92]. The spectrum of the $c$-ZSM-22 sample shows a larger contribution of internal silanols and thus higher amount of such defects. As expected for the $m$-ZSM-22, having a lower $\mathrm{Si} / \mathrm{Al}$ ratio, the intensity of the $3600 \mathrm{~cm}^{-1}$ band is larger compared to the $c$-ZSM- 22 . However, the concentration of Brønsted sites is the same for both samples (Table 3). This suggests that there are stacking faults in the $m$-ZSM-22 sample, preventing the diffusion of the probe molecule through the unidirectional channel system. The frequency shift of about $\Delta v=325 \mathrm{~cm}^{-1}$ seen for the Brønsted band due to the $\mathrm{OH}-\mathrm{CO}$ interaction upon $\mathrm{CO}$ adsorption (Fig. S8a,c) is comparable with other zeolites and suggest an intermediate Brønsted acidity [91]. Noteworthy, the BAS band was not completely eroded for the $m$-ZSM-22 sample, even after high $p(\mathrm{CO})$ dosage (Fig. S8c). Also, the spectra of the $\mathrm{OH}$-stretching region shows that not all the BAS are consumed after adsorption of pyridine (Fig. S10a), suggesting that some of the BAS are not accessible for $m$-ZSM-22.

\subsection{Impact of the desilication treatments}

As it has been highlighted, the extent of crystal dissolution upon desilication and the resulting pore structures and acidity initially depend on the morphology and composition of the starting zeolite [19]. The complexity and interdependency of all the parameters that influence the desilication makes the interpretation of the results complex. Furthermore, the desilication treatment conditions may notably influence the mesopore formation $[19,26,27]$. 


\subsubsection{Structure, composition and morphology}

The crystallinity of the starting zeolites was maintained after the desilication, as confirmed by the PXRD diffractograms shown in Fig. S1 The cristobalite phase was only evident after the $a t$ and $t b a$ treatments of the commercial sample and was effectively removed upon the rest of treatments. It may be noted that the $c$-ZSM-22-ats2 sample displays a peak at 11.7 degrees, which is indicative of an undefined dense phase. The yield of the treatments (defined as 100 - weight loss \% after the treatments) and the $\mathrm{Si} / \mathrm{Al}$ ratio of the treated samples are reported in Table 2 . The $m$-ZSM-22 zeolite with higher acid site density is expected to be more difficult to desilicate than $c$-ZSM-22 due to the aforementioned protective effect of the framework $\mathrm{Al}$ [17]. However, a similar extent of Si extraction and crystal dissolution was obtained independently of the starting material. Overall, the same desilication approach led to similar yields for both the commercial and the in house prepared samples. As expected, the solid yield is reduced with the severity of the treatment for each desilication series, i.e. the more severe treatments led to more extensive crystal dissolution.

$\underline{\text { Figure } 6}$ presents the variation of the $\mathrm{Si} / \mathrm{Al}$ ratio as a function of the $\mathrm{NaOH}$ molarity for the desilicated samples. The dashed curve indicates the Si/Al variation when only $\mathrm{NaOH}$ was used as desilication agent. As expected from the similar dissolution rates, similar reduction of the $\mathrm{Si} / \mathrm{Al}$ ratio is systematically observed for the commercial and in house prepared materials with increased $\mathrm{NaOH}$ concentration. After a drastic reduction of the initial $\mathrm{Si} / \mathrm{Al}$ ratio upon the mild treatment, the severe $\mathrm{NaOH}$ solution led to a lower extent of decrease, suggesting that most of the $\mathrm{Si}$ atoms can be efficiently removed from the zeolite already with the less severe alkaline treatment. This leveling off suggests an upper limit for Si extraction. When the additional desilication agents 
were added to the alkaline solution, the $\mathrm{Si} / \mathrm{Al}$ ratio trends deviate from the reference curve, and appears to do so in a non-predictable manner. As an example, for the low concentration surfactant-assisted approach (atsl), for both starting materials, this treatment leads to $\mathrm{Si} / \mathrm{Al}$ ratios above the reference curve. On the other hand, the effect seen at the higher concentration (ats2) is the opposite. With respect to the tba treatment, the reduced $\mathrm{Si} / \mathrm{Al}$ ratio and the higher yields as compared with the other processes are indicative of a more selective Si removal. Si dissolution was more effective than in $\mathrm{NaOH}$ solution, in contrast with previous works on ZSM-5 $[72,75,76]$. The increase of the temperature during the process had little impact beyond slightly more Si extraction. The data listed in Table 2 show a reduction in the $\mathrm{Si} / \mathrm{Al}$ ratio compared to the starting material for all samples, clearly showing that $\mathrm{Si}$ was preferentially removed. A deeper analysis can be reaced by predicting the $\mathrm{Si} / \mathrm{Al}$ by multiplication of the sample yield with the $\mathrm{Si} / \mathrm{Al}$ ratio of the starting zeolite, therebye assuming that only $\mathrm{Si}$ is removed. Table $\underline{2}$ shows that the predicted $\mathrm{Si} / \mathrm{Al}$ values are lower than the ones determined by elemental analysis for the samples treated with $\mathrm{NaOH}$, whereas similar or higher predicted $\mathrm{Si} / \mathrm{Al}$ ratios were obtained for the surfactant-assisted and $\mathrm{TBAOH} / \mathrm{NaOH}$ treated samples, respectively. Thus, it appears that for the $\mathrm{TBAOH} / \mathrm{NaOH}$ treated samples in particular, Al may also be extracted from framework positions upon desilication, This partial loss of $\mathrm{Al}$ results in a $\mathrm{Si} / \mathrm{Al}$ ratio above the predicted value. In should also be considered that in addition to being extracted from the crystals, $\mathrm{Al}$ can be redistributed as EFAl species, which will not be seen as a change in $\mathrm{Si} / \mathrm{Al}$ ratio [17]. Previous work has identified the EFAl species as $\mathrm{Al}^{+3}$ (Lewis), oxoaluminium $\mathrm{AlO}^{+}, \mathrm{Al}(\mathrm{OH})_{2}{ }^{+}$or $\mathrm{Al}(\mathrm{OH})^{2+}$ cations, or neutral species such as Al-OH species or Al-OH species [17, 31, 90, 93, 94]. In addition, the desilication treatments may lead to other structural effects, such as T-OH groups formed due to the lack of a chemical bonding between two vicinal $\mathrm{TO}_{4}$ 
tetrahedra, or hydroxyl silanol nests as extra-framework Si species (EFSi) when a T atom is missing [17, 90, 95-97].

A morphology investigation using SEM indicates that different zones of the crystal were preferentially dissolved during desilication depending on the starting material. The external surfaces of the thinner crystals of $m$-ZSM-22 were more selectively affected, resulting in eroded nanoparticles with high exposed surface area. In contrast, for $c$ ZSM-22, the inner zones of the particles were the most affected, resulting in the creation of intra-crystalline mesopores. SEM micrographs of the desilicated samples show that the rod-like agglomerates of $c$-ZSM-22 are transformed into fragmented nanocrystals of irregular size and shape after at and ats treatments (Fig. S2c-f), whereas the tba treatment did not result in no apparent modification of the parent crystal morphology at the magnification achieved by SEM (Fig. S2g). The in house prepared material was differently affected: all the desilication treatments led to dissolution of the outer surface of the needle-shaped crystals, which became shorter and thinner (Fig. S3). TEM images of the desilicated samples are shown in Fig. 1 and Fig. S4. The bright areas in the micrographs are interpreted as the removal of siliceous zones inside the zeolite crystals. TEM images of the desilicated commercial zeolite clearly reveal the presence of mesopores. The less severe $\mathrm{NaOH}$ treatment resulted in a partial dissolution of siliceous areas along the fragmented crystal boundaries, with a lower contribution of intra-crystalline mesopores (Fig. S4a), whereas pores of different sizes are also created inside the crystal after treatment with high concentration, as shown in Fig. 1b. The combination of crystal roughening and mesopore creation agrees with the higher extent of framework dissolution observed for this sample (Table 1 and Fig. 6). The addition of surfactant to the alkaline solution resulted in smaller and more defined mesopores within the crystals of the commercial sample, and they appear to be evenly distributed 
along the crystal (Fig. 1c and Fig. S4b). The crystal edges appear to be less affected in this case, confirming that the addition of the CTAB surfactant caused a more controlled mesopore generation. Intra-crystalline mesopores are also created after the tba treatment of the commercial sample. The low temperature treatment generated less mesopores, mainly located on the boundaries of the crystal (Fig. S4c), whereas higher temperature led to intra-crystalline mesopore generation in the crystal (Fig. 1d), in agreement with the higher degree of crystal dissolution seen for this sample (Table 2 and Fig. 6). The pores appear to be larger than the ones generated by the surfactant-aided method. Overall, the SEM and TEM results indicate that the commercial $c$-ZSM-22 crystals are big enough to allow the formation of intra-crystalline mesopores. The TEM images suggest that for the surfactant-assisted treatment, mesopores are more effectively created via selective dissolution of siliceous zones from the bulk crystal [14, 35]. For the at and tba approaches, mesopore formation by preferential dissolution along boundaries and framework defects within the crystal cannot be dismissed $[31,35,38$, 92].

The same desilication methods did not result in appreciable mesopore creation for the thinner needle-like crystals of the $m$-ZSM-22. Instead, the external surface of the original nanorods was roughened after the treatments. Even though no large differences are found between the low and high concentration $\mathrm{NaOH}$ treatments, the crystals seem to be more affected and fragmented after the more severe treatment (Fig. 1f). When the CTAB was added to the solution, the concentration appears to influence the morphology. The less concentrated treatment led to a similar effect as the alkaline method i.e. the needle-shaped nanocrystals appear to shrink in size (Fig. S4e). However, the original needles are no longer apparent after the treatment with higher alkaline concentration. Instead, the crystal boundaries are drastically dissolved and nanoclusters 
with irregular shape are formed (Fig. 1g). This is in accord with the higher loss of material seen for this sample (Table 2 and Fig. 6). The two tba treatments had a similar and minor effect on the in house prepared zeolite. The needles become slightly narrower after the treatment at higher temperature (Fig. 1h). Generally, it should also be kept in mind that the mode of crystal dissolution could also be influenced by an uneven distribution of the $\mathrm{Al}$ : a lower $\mathrm{Al}$ concentration on the external surface of the crystal would result in a lower protective effect against the $\mathrm{Si}$ extraction in this zone as proposed by Dessau et al. [98] and demonstrated later [75, 76] for MFI zeolites.

\subsubsection{Porous properties}

Figure 2 shows the $\mathrm{N}_{2}$ isotherms of the parent and desilicated samples. The numerical output from these experiments are listed in Table 2. The microporous type I isotherm of the parent $c$-ZSM-22 zeolite is transformed into type II - IV isotherms (Fig. 3a-c) after desilication, which are characteristic of mesoporous materials [88]. The same holds for the treated $m$-ZSM-22 samples (Fig. $2 \mathrm{~g}-\mathrm{i}$ ). Contrary to previous works [58], the microporosity of the commercial zeolite was generally unaffected by the desilication treatments, as indicated by the similar $\mathrm{N}_{2}$ uptake at low partial pressures of the isotherm. Only the $c$-ZSM-22-tba2 sample shows a $\sim 38 \%$ loss in micropore volume. As the crystallinity is intact (Fig. S1), this reduction is most likely caused by extraframework species redeposited on the pore entrances, see below [54, 56, 58]. With respect to the series based on the in house prepared material, the microporosity was drastically reduced after the surfactant-assisted and the high temperature tba process, by as much as $60-80 \%$. Again, as the crystallinity is intact, this implies the presence of blocking species. However, the rest of the treatments resulted in an increased micropore volume. 
For an easier tracking of the extensive amount of data, the variation in pore volume and surface area for the different desilication treatments is schematically presented in Fig. 4. We will use $S_{\text {ext }}$ and $V_{\text {ext }}$ to refer to the surface area or volume of the pores larger than the micropore size $(>2 \mathrm{~nm})$. These quantities are generally correlated, but with substantial spread (linear regression of the entire dataset gives a positive slope, but a quite poor value $\mathrm{R}^{2}=0.14$ in Fig. S7b). Generally, $S_{\text {ext }}$ and $V_{\text {ext }}$ were intact or substantially increased for all the samples after the various treatments. A reduction of the total surface area $\left(S_{B E T}\right)$ is observed for the samples with reduced microporosity. Micropore volume and $S_{B E T}$ follow each other quite closely $\left(\mathrm{R}^{2}=0.87,27\right.$ data points), as shown in Fig. S7a. For the samples with reduced total surface area/micropore volume, the $S_{\text {ext }}$ was barely affected or even increased. For the at samples (those treated with only $\mathrm{NaOH}), S_{\text {ext }}$ was close to doubled compared to the starting materials, and the increase in did not depend on the $\mathrm{NaOH}$ concentration (Table 2 and Figure 4a,c). Such an increase in external surface was expected, owing to the dissolution and fragmentation of the crystals, leading to smaller particles with higher exposed external surface $[27,54$, 58]. A slightly different picture becomes evident when inspecting the $V_{\text {ext }}$ values, a manifestation of the relatively poor correlation between $S_{\text {ext }}$ and $V_{\text {ext. }}$ The severe alkaline treatment of the commercial material caused a significantly higher increase in $V_{\text {ext }}$ compared to all other at samples (Fig. 4b), probably as a consequence of the broad mesopore size distribution depicted in the BJH plot (Fig. 3a). The non-uniform distribution of mesopores indicates that the framework was irregularly dissolved. These mesopores are still small enough to be accommodated within the ZSM-22 particles and confirms the microscopy observations. As mentioned in Section 3.1., the zig-zag shape observed at larger radios in the BJH plots for the in house prepared zeolites (Fig.3d-f 
and Fig. S6d-e) and for the commercial samples treated with $\mathrm{NaOH}$ (Fig. 3a) do not represent true mesoporosity but is considered an artifact of the analysis.

Turning to the ats samples, a similar increase of $S_{\text {ext }}$ was found for the ats 1 treatment irrespective of the starting material. However, the higher concentrated process resulted in a remarkable increase of the $S_{\text {ext }}$ of the commercial zeolite (Fig. 4a), but virtually no change was seen for the in house prepared zeolite (Fig. 4c). For the treated commercial samples, an increase in $V_{\text {ext }}(\underline{\text { Fig. }}$ 4b) was observed. Clearly, the framework dissolution was in this case accompanied by the creation of defined mesopores of 2-3 $\mathrm{nm}$ in size as seen in the BJH pore size distribution (Fig. 3b). It has been suggested that these intracrystalline mesopores could be formed by the reassembly of dissolved zeolite fragments upon the surfactant treatment $[54,67-69]$ and that this process is initiated around small irregular pores (defects), as already seen for the parent $c$-ZSM-22 sample. On the other hand, no appreciable mesopores were evidenced in the $\mathrm{BJH}$ plots for the in house prepared samples prepared by the surfactant-assisted route (Fig. 3e). Surprisingly, the higher dissolution evidenced for the m-ZSM-22-ats2 crystal (Fig. 1g) was not reflected in an increase in $S_{\text {ext }}$. It seems very difficult to create large mesoporosity in $m$-ZMS-22 because of the very small thickness of the crystal rod. The size of the crystal is 0.05 microns (i.e. $50 \mathrm{~nm}$ ). Then, there is not enough room for making large pores. Indeed, pores of $10 \mathrm{~nm}$ quoted for $20 \%$ of the total thickness.

Only minor variation in $S_{\text {ext }}$ is observed when the $c$-ZSM-22 sample is treated with the $\mathrm{NaOH} / \mathrm{TBAOH}$ solutions. However, the $\mathrm{BJH}$ pore size distribution and the increase in $V_{\text {ext }}$ evidence the formation of larger mesopores at higher temperature (Fig. 3c), in agreement with the voids previously seen in the TEM micrographs (Fig. 1d). The smaller increase in $V_{\text {ext }}$ achieved compared to the alkaline and the surfactant-assisted method is associated with the limited uptake $\mathrm{N}_{2}$ increase at high partial pressures (Fig. 
2c). The broad mesopore size distribution confirms the presence of mesopores which are larger ( 2 to $10 \mathrm{~nm}$ in size) than the ones created by the surfactant-assisted treatment, although they add up to a smaller created mesopore volume (Fig. 3c). Such defined mesopores are likely a result of the pore directing character of the $\mathrm{TBA}^{+}$ions $[72,75$, 76, 79]. For the in house prepared set, the high temperature treatment resulted in an increase of the $S_{\text {ext }}$, whereas the $V_{\text {ext }}$ was reduced, suggesting a larger dissolution of the outer crystal surface. The presence of extra-framework species, apart from blocking the micropores, might inhibit the creation of mesopores in this case. In contrast to the commercial set, no mesopores are evident in the $\mathrm{BJH}$ pore size distribution plots after the $t b a$ treatments of the in house prepared sample (Fig. $3 \mathrm{e})$.

The insets of Fig. 2 highlight particular regions for each isotherm. The shape and type of the created mesopores for the commercial series can be estimated from the shape of the hysteresis loop [85, 88]. The at samples show limited H3-type hysteresis (Fig. 2a), characteristic of agglomerates of particles forming slit-shaped pores with non-uniform size or shape. More evident hysteresis is observed for the commercial sample treated with $\mathrm{NaOH}$ and $\mathrm{CTAB}$ (Fig. 2b), and this is distinctive of more uniform and possibly ink-bottle shaped mesopores. The commercial sample treated with $\mathrm{NaOH} / \mathrm{TBAOH}$ at high temperature shows close to H1-type hysteresis (Fig. 2c), which suggests the formation of nearly cylindrical, uniformly sized pores.

The majority of the treatments generally improved the porous properties (higher micropore and mesopore volumes/surfaces) of both starting materials. However, severe reductions of total surface area were also encountered. This is further discussed in section 3.3. This analysis also confirmed the remarkable impact of the starting crystal morphology on the mechanism of mesopore generation: even though similar extent of crystal dissolution was observed, intra-crystalline mesopores were effectively created 
only within the rod-like commercial crystallites, whereas the thinner dimensions of the needle-shaped particles of the in house prepared zeolite represented an obstacle for the development of intra-crystal mesoporosity. The lower microporosity of the $m$-ZSM-22 sample could be responsible of the lack of intra-crystalline mesoporosity formation upon alkaline treatment, because bases and/or silicates species cannot diffuse freely through the unidirectional channel system.

The actual mesoporosity seen in the TEM micrographs and estimated in the BJH pore size distribution plots for the commercial zeolites treated with $\mathrm{CTAB} / \mathrm{NaOH}$ or $\mathrm{TBAOH} / \mathrm{NaOH}$ should also be reflected as increased external surface or external volume. Fig. S7c, d shows the $S_{\text {ext }}$ and $V_{\text {ext }}$ values for the samples showing clear mesopores in TEM and BJH pore size distribution together with the rest of samples. Contrary to was expected, $S_{\text {ext }}$ and $V_{\text {ext }}$ of the cited samples do not stand out from the rest of samples, suggesting a minor influence of the actual mesoporosity in the external surface or volume. Only the material treated with the severe surfactant-assisted method (c-ZSM-22-ats2) showed a marked increase in $S_{\text {ext }}$ as a consequence of the created mesopores. As a technical point, we note that $S_{\text {ext }}$ correlates more clearly with actual mesoporosity as seen in the BJH plots and TEM than $V_{\text {ext }}$.

\subsubsection{Acidity}

The effect of the desilication treatments on the acidity has been qualitatively investigated by IR spectroscopy. For this purpose, one sample of each desilication approach was selected: $c$-ZSM-22-at1, $c$-ZSM-22-ats1 and $c$-ZSM-22-tba2 for the commercial series; and $m$-ZSM-22-at1, $m$-ZSM-22-ats $1, m$-ZSM-22-tbal for the in house prepared set. 
Figure 5 shows the FT-IR spectra of the protonated desilicated samples activated at 450 ${ }^{\circ} \mathrm{C}$ under vacuum. Essentially, the band associated with the BAS at $3600 \mathrm{~cm}^{-1}$ was slightly reduced after the desilication treatments of the commercial zeolite, whereas a significant increase in the isolated silanol band at $3745 \mathrm{~cm}^{-1}$ is readily observed for all the desilicated samples (Fig. 5a). A tendency towards the reduction of strong Brønsted sites concentration and parallel formation of weaker acid sites upon desilication was previously demonstrated for TON zeolites [56-58]. On the other hand, a rather small variation in the intensity of the BAS band has also been shown for desilicated MFI zeolite [75]. Our results for the commercial material agree better with the latter observation, i.e. that the concentration of Brønsted sites do not substantially change upon desilication. The Si/Al decreases upon desilication (Table 2). Then, desilicated samples must give higher acid site concentration if $\mathrm{Al}$ remains the same. This indicates that there is EFAl species in desilicated materials. The larger concentration of external silanol groups is readily associated with the increment of $S_{\text {ext }}$ and $V_{\text {ext }}[21,31,35,56-$ 58]. As an exception, the c-ZSM-22-ats1 sample presents a clear decrease in the intensity of the BAS band. In addition, a sharp increase of the isolated silanols band at $3745 \mathrm{~cm}^{-1}$ is seen. The surfactant-assisted method resulted in a greater number of mesopores and consequently, more exposed external surface was created, where more isolated silanol groups can be located. Conversely, the band at $3745 \mathrm{~cm}^{-1}$ increased less after the $a t$ and $t b a$ treatments, in line with the notion that less created mesopore surface can be linked to a lower contribution of external silanol groups.

Focusing on all the desilicated in house prepared zeolites, again a general reduction of the intensity of the $3600 \mathrm{~cm}^{-1}$ band and a loss of the Brønsted acidity is evidenced (Fig. $\underline{5}$ b). Previous qualitative FTIR experiments using pyridine as probe molecule attributed the decrease in Brønsted acidity upon desilication of TON zeolites to a micropore 
blockage due to the presence of extra-framework residues, such as poorly alumina-like species on the external surface of the crystal [54, 56-58]. Our results indicate that the desilication process also removed $\mathrm{Al}$ from framework $\mathrm{Si}(\mathrm{OH}) \mathrm{Al}$ positions, which may be extracted from the crystal, or yielding to EFAl species. Together with the microscopy, the results suggest that most of the framework $\mathrm{Al}$ is extracted from external acidic positions, reducing the protecting effect on neighboring $\mathrm{Si}$ atoms and thus promoting the desilication in external surface areas. As for the commercial series, the band associated with isolated silanol groups was increased after the desilication treatments of the in house prepared material, but to a lesser extent. Specifically, the at and tba treatments led to a slight increase of the external silanols band, whereas the ats treatment resulted in a narrower and more intense band at $3745 \mathrm{~cm}^{-1}$, as a result of larger contribution of silanols located at the created external surface on the more dissolved crystal boundaries of this sample.

Interestingly, the absorbance seen at wavenumbers ranging from $3650 \mathrm{~cm}^{-1}$ to $3735 \mathrm{~cm}^{-}$ 1, varies with respect to the starting material. The commercial material has a substantially higher absorbance in this region relative to the in house prepared material. Bands in this region can be associated to the presence of $\mathrm{OH}$ groups interacting weakly with $\mathrm{Si}$ in different environments inside the zeolite framework [95]. Further, the raised baseline seen for the in house prepared zeolite in this region is no longer noticeable for the desilicated samples (Fig. 5b). Conversely, the intensity was not noticeably reduced, or even increased, after the desilication treatments of the commercial zeolite. Only the tba treatment resulted in a decrease of the intensity in the lower frequency range of this region (Fig. 5a). We propose that a rationalization of this behavior might found in the TEM analysis. The shrinking of the thinner, aggregated crystals of the in house prepared parent zeolite after desilication might also have cleaned up the weaker interacting 
silanols, which might be ascribed to defects in the micropores near external crystal zones. On the other hand, desilication of the larger commercial crystals did not remove the weaker silanols, but resulted in a larger contribution of defects, which might be located on the created mesopore surface inside the zeolite crystal.

The accessibility and the acid strength were investigated with FT-IR combined with CO and pyridine adsorption. From the CO adsorption data (dotted spectra in Fig. S8), a similar frequency shift of the BAS band is observed for all the treated samples and both starting zeolites, indicating that the acid strength is unaffected by the desilication treatments. Some particular features are now described. The BAS band at $3600 \mathrm{~cm}^{-1}$ was not completely eroded for the $m$-ZSM-22-at1 and m-ZSM-22-tbal samples, and was practically unaffected for the $m$-ZSM-22-ats 1 sample, even at high $\mathrm{CO}$ dosage. Also, the Brønsted acid sites were not fully accessible to pyridine for these samples either (see Fig. S10). Clearly, the limited access to the BAS is most evident for the surfactant-assisted treated in house prepared samples (Fig. S10d). The band associated with the external silanol groups of $c$-ZSM-22-tba2 was almost unaffected upon CO adsorption, but is reduced for the rest of treated samples (Fig. S8a). This observation implies that the $\mathrm{CO}$ was not able to access all the silanols sites, part of them might be located in non-open mesopores.

A distinct shoulder of the $3280 \mathrm{~cm}^{-1}$ band is evidenced at $3400 \mathrm{~cm}^{-1}$ for all the desilicated samples (asterisk in Fig. S8). Spectroscopic studies over desilicated ZSM-5 zeolites ascribed this shoulder to internally located $\mathrm{OH}$ groups of EFAl hydroxides [21, 31]. The shoulder at $3400 \mathrm{~cm}^{-1}$ have been also attributed to the presence of weaker sites, to the heterogeneity of $\mathrm{OH}$ groups in the zeolite framework or to inhomogeneous distribution of $\mathrm{Al}^{+3}$ ions $[91,95]$. However, no band associated with extra-framework $\mathrm{Al}^{+3}$ species was observed at $2230 \mathrm{~cm}^{-1}$ in the $\mathrm{CO}$ stretching region for the treated 
samples (Fig. S8b,d). This apparently indicates that the amount of EFAl species is negligible. However, the absence of $\mathrm{CO}$ interactions with $\mathrm{EFAl} \mathrm{Al}^{+3}$ species could also be in accordance with the blockage of the one dimensional channels of ZSM-22 by small $\mathrm{Al}$ clusters or other extra-framework species, giving rise to inaccessible $\mathrm{Al}$ sites [91].

\subsection{Effect of the acid washing}

The impact of the sequential acid leaching is investigated in this section. The desilicated and acid treated samples consist of a high purity TON phase, as shown in PXRD diffractograms (Fig. S1). The cristobalite impurity seen for the commercial tba samples was effectively removed with the acid washing.

Table 2 shows that the acid treatments returned the $\mathrm{Si} / \mathrm{Al}$ ratio to the original value of $\sim 49$ for the commercial zeolites and $\sim 38$ for the in house prepared materials. These results support the suggested process of $\mathrm{Al}$ extraction and redeposition as EFAl species upon desilication. The EFAl species are then removed after the sequential acid treatment, restoring the $\mathrm{Si} / \mathrm{Al}$ ratios [58]. The acid washing of the surfactant treated in house prepared samples did, however, not result in a variation of the $\mathrm{Si} / \mathrm{Al}$ ratio, suggesting that either no EFAl species were generated upon this treatment, or that the acid treatment was not capable of dissolving them.

The acid washing was expected to have a marked influence on the porosity of the samples for which micropore volume was reduced upon desilication $[37,58,64]$. The analysis of the microporosity reveals two features: firstly, the acid washing of the samples treated with tba at high temperature restored the original microporosity for both commercial and in house prepared materials (Table 2), as seen by the increase in $\mathrm{N}_{2}$ uptake (insets in Fig. 2f,1) and the $S_{B E T}$ values in Fig. 4. This suggests that the acid 
treatment successfully removed the redeposited extra-framework species. Conversely, the micropore volume of the surfactant treated in house prepared samples was not affected after the acid treatment (Table 2 and Fig. $2 \mathrm{k}$ ). Together with the invariance of the $\mathrm{Si} / \mathrm{Al}$ ratio, these results indicate that the acid leaching was not sufficient to remove the blocking species in this case. In contrast to the three-dimensional MFI framework type, which exhibits relatively large pores and connected channels allowing a better elimination of the potentially blocking extra-framework species generated upon desilication, the ellipsoidal one-dimensional pore system of the ZSM-22 zeolite prevents these species to diffuse out of the framework $[58,91]$.

The acid washing affected the $S_{\text {ext }}$ and $V_{\text {ext }}$ of the desilicated samples to a lesser extent than the desilication treatments. The $S_{\text {ext }}$ was increased after the acid washing of the commercial at or tba treated samples, but unchanged after the acid leaching of the surfactant-assisted treated zeolites (Fig. 4a). The acid treatment resulted in slightly increased $V_{\text {ext }}$ with respect to the desilicated counterparts. Noteworthy, the acid washing of the mild alkaline treated sample (c-ZSM-22-at1-HCl) caused a remarkable higher increase of the $V_{\text {ext }}$ compared to the rest of acid washed samples (Fig. 4b). For the in house prepared series, the main findings were a significant reduction of the $V_{\text {ext }}$ after the acid washing of the ats samples and a reduction of the $S_{\text {ext }}$ and increasing of $V_{\text {ext }}$ for the m-ZSM-22-tba2-HCl sample (Fig. 4c,d). The exclusive acid washing of the fresh ZSM22 commercial sample ( $c$-ZSM-22-HCl) did not have any remarkable influence on the textural properties (Table 2 and Fig. S5).

According to the FT-IR spectra of the dehydrated acid washed samples presented in Fig. $\underline{5}$, the distribution of surface sites of the desilicated samples was barely affected by the acid washing. The BAS band at $3600 \mathrm{~cm}^{-1}$ was essentially unmodified. These findings indicate that removal of the redeposited EFAl species occur without a marked alteration 
of the framework Al concentration and thus the strong Brønsted acidity. The band associated with the isolated silanol groups at $3745 \mathrm{~cm}^{-1}$ generally increased slightly after the acid leaching, in agreement with the $S_{\text {ext }}$ variation for the commercial desilicated and acid treated samples (Fig. 4a), but increased significantly after the acid treatment of the commercial alkaline treated sample (c-ZSM-22-atl-HCl), as shown in Fig. 5a). This indicates that the acid washing contributes to clean up not only the strong acid sites, but also to some extent the silanol defects.

Comparable acid strength was observed for the acid washed materials with respect to the desilicated counterparts, as indicated by the similar frequency shift of the BAS band after CO dosing in Fig. S8a,b. From Fig. S8a it is clear that the inaccessible silanols of the $c$-ZSM-22-tba2 sample are now interacting with the CO molecule, confirming that the blocking species are successfully removed with the acid washing. With respect to the acid treated $m$-ZSM-22-atl- $H C l$ and $m$-ZSM-22-tbal-HCl samples, the erosion of the $3600 \mathrm{~cm}^{-1}$ band and the creation of a high intensity band centered at $3275 \mathrm{~cm}^{-1}$ implies that the rather inaccessible BAS of the desilicated counterparts are now accessible to the $\mathrm{CO}$ molecule after the acid treatment. These findings are confirmed by the erosion of the BAS band seen when pyridine is adsorbed on the $m-\mathrm{ZSM}-22-a t 1-\mathrm{HCl}$ sample (Fig. S10c). The Brønsted sites were still inaccessible after the acid washing of the $m$-ZSM-22-ats 1 sample, as confirmed by the invariance of the absorbance band at $3600 \mathrm{~cm}^{-1}$ after pyridine dosing (Fig. S8e). This confirmed the reduction in micropore volume observed for this sample.

\subsection{Interdependence between composition, acidity and porosity}

Owing to the simultaneous variation of several parameters during the desilication and the sequential acid washing, we assume a synergetic effect of the studied properties. In 
the present section we attempt to identify potential correlations between composition, acidic, and porous properties. It appears evident that desilication not only causes dissolution of the crystal, but also a reorganization of the $\mathrm{Al}$ species.

The acidity of the selected samples is investigated quantitatively by pyridine adsorption monitored by FT-IR spectroscopy. The concentration of Brønsted and Lewis acid sites was quantified by the integration of the bands at $1545 \mathrm{~cm}^{-1}$ and $1455 \mathrm{~cm}^{-1}$ [99]. Pyridine has a kinetic diameter of $5.7 \AA$, similar to the maximum channel opening of ZSM-22 $[50,52,53]$, and represents a sterically demanding molecule. It has been shown that the access to BAS was drastically blocked only for the $m$-ZSM-22 and the $m$-ZSM-22-ats 1 samples (Fig. S10). Also, a small fraction of BAS seems not to be accessible to the pyridine for $m$-ZSM-22-at1 and $m$-ZSM-22-tbal samples, suggesting that the unaccessible sites are located in occluded mesopores [31].

The amount of acid sites was correlated with the total Al concentration. Table 3 presents the Brønsted (BAS), Lewis (LAS) acid site and BAS+LAS concentration, together with the total amount of $\mathrm{Al}$ calculated as from elemental analysis. The last column indicates the percentage of the $\mathrm{Al}$ forming acidic sites. The data is graphically represented in Fig. $\underline{7} \mathrm{a}$, where the dotted line represents the situation where all the $\mathrm{Al}$ atoms form acidic sites. The deviation of the parent $c$-ZSM-22 zeolite from the dotted line together with the low contribution of Lewis acidity (ratio BAS:LAS of 8:1), suggests that non-acidic Al species are already present in the starting material. Clearly, the $m$-ZSM-22 parent material also deviates from the reference line, but the limited access to the BAS needs to be considered in this case.

Two different features are readily observed for the treated samples. On one hand, the concentration of acid sites is lower than the total $\mathrm{Al}$ concentration for the desilicated samples (solid points in Fig. 7), but not more so than the starting materials. The 
desilication procedures lead to approximately a four fold increase of LAS relative to the corresponding parent material, with the exception of two samples: for $m$-ZSM-22-tbal the contribution of LAS was doubled and for $m$-ZSM-22-ats 1 no remarkable increase was seen (Table 3 and Fig. 7b). The results suggest a conversion from Brønsted acid sites into Lewis acid sites upon desilication. On the other hand, the acid washed samples follow closely the dotted line (open symbols in Fig. 7), showing a very similar concentration of acidic sites and total Al. The m-ZSM-22-ats1-HCl sample deviates from this behavior. However, the general trend is that the acidity is recovered after acid washing. At the same time, the Lewis acidity is reduced with respect to the desilicated analogues (Fig. 7b), supporting the removal of EFAl species by the acid leaching and linking EFAl species to LAS.

The acidity modifications during the treatments should be related to the porosity. It is generally accepted that the LAS are associated with EFAl species, which might be redeposited on the external zeolite surface or mesopore surface after desilication [19, $31,58,65]$. In our study, these species can be assumed to be redeposited on the external surface created for the in house prepared crystals or on the mesopore surface created for the commercial samples, before being washed out by the acid treatment. Figure $7 \mathrm{~b}$ shows the correlation between LAS and external surface area. For the desilicated samples, the amount of LAS reached a level where any further increment in $S_{\text {ext }}$ imply no further gain in Lewis sites. This suggests that the formation of LAS is limited by another parameter, such as the initial number $\mathrm{Al}$ species in non-framework positions. A nearly linear correlation was found for the LAS concentration and the $S_{\text {ext }}$ for the acidwashed samples, indicating strongly that the remaining LAS were located on the created external (or mesopore) surface. 
At this point, a question arises about the origin of the EFAl species and their redistribution upon desilication. The BAS concentration should increase since Si/Al decreases upon desilication. In our case, however, the process over the commercial sample occurs without a pronounced alteration of the Brønsted acidity, which suggests a transformation of Brønsted to Lewis acid sites. As seen in Fig. 7, there is a fraction of non-acidic species already present in the starting material. We speculate that these remain after desilication, and that part of them also generate Lewis acid sites upon desilication. The extra-framework species, including a substantial part of Lewis groups, are eventually removed by acid washing. Regarding the in house prepared series, both contributions of framework $\mathrm{Al}$ in $\mathrm{Si}(\mathrm{OH}) \mathrm{Al}$ groups and $\mathrm{Al}$ species already present in the starting material, could contribute to the formation of Lewis species.

A more detailed analysis highlights particular features for the samples listed in Table 3. The $c$-ZSM-22-tba2 sample shows an increase of concentration of Brønsted sites, even though no variation was observed in the intensity of the BAS band in the activated spectra with respect to parent material (Fig. 5a). This was unexpected since all Brønsted sites were available for the starting and this treated sample. The loss in Brønsted acidity after acid washing for the $c$-ZSM-22-tba2 and the $m$-ZSM-22-tbal sample was not expected. A possible explanation could be a redistribution of the remaining EFAl species hampering the pyridine to reach the Brønsted sites. Also, Fig. S10g shows that some of the BAS are still no accessible to pyridine for latter sample. The particular case of the surfactant-assisted treated $m$-ZSM-22-ats 1 samples is highlighted with red points in Fig. 7. The lower contribution of acidic sites was a result of the limited accessibility for pyridine due to micropore blockage. The rather low contribution of LAS for both surfactant- and acid-treated samples suggests that other extra-framework species than $\mathrm{Al}^{+3}$ (Lewis sites) cause the hindrance effect. 


\section{Conclusions}

In this contribution we investigate the impact of three different desilication approaches, i.e. $\mathrm{NaOH}$ treatment, treatment using mixtures of $\mathrm{NaOH}$ with $\mathrm{CTAB}$ and using mixtures of $\mathrm{NaOH}$ with TBAOH, on two different ZSM-22 zeolite samples. Even though the introduction of mesopores by desilication is dependent on many parameters, such as acid site density and $\mathrm{Al}$ distribution, defects and treatment conditions, the crystal morphology of the starting zeolite appears to be the dominant parameter which influences the mesopore generation. Mesopores were effectively created within the rodlike commercial crystallites, whereas the thinner dimensions of the needle-shaped particles of the in house prepared zeolite represent an obstacle for an intra-mesopore creation. Instead, the boundaries of the needle crystals were preferentially affected, resulting in fragmented nanoparticles with higher external surface area.

This contribution opens a route for an effective creation of intra-crystalline mesopores within the one-dimensional pre system of ZSM-22, since the alkaline, surfactantassisted or combined $\mathrm{NaOH} / \mathrm{TBAOH}$ desilication methods differently influenced the shape and size of the created mesopores for the commercial series. The conventional alkaline treatment with $\mathrm{NaOH}$ resulted in both roughening of the crystal surface and creation of non-uniform mesopores, whereas when CTAB or TBAOH agent was added, a well-defined mesopore size distribution is obtained. The surfactant-assisted treatment led to the formation of smaller mesopores of $2 \mathrm{~nm}$ in size, while larger pores of about 3 $8 \mathrm{~nm}$ in size are generated when $\mathrm{TBAOH}$ is added to the alkaline solution. The sequential acid washing generally resulted in increased the micropore volume with respect to the desilicated samples. Elemental analysis showed that EFAl species were generated upon the desilication treatments, which are eventually removed by the 
subsequent acid treatment. The acidity studied by FTIR demonstrated that the generation and redistribution of extra-framework species occurs without a marked modification of the Brønsted acidity, whereas the concentration of surface silanol hydroxyl groups is increased after the treatments. The comparison between the total Al concentration and the amount of $\mathrm{Al}$ forming acidic sites shows that the acidity was recovered after the acid washing and suggest that non-acidic Al species are already present in the starting materials and may have a role in the formation of both Lewis and EFAl species upon desilication. Despite the large number of samples studied in this investigation, we do not succeed in arriving at a predictable procedure for mesopore introduction, and general trends are always accompanied by inexplicable deviations. Thus, a better knowledge of the complex transformations involved during the desilication process is still required to provide rational tools for a better control of the desilication. This constitutes a particular challenge for one-dimensional systems such as ZSM-22 zeolite, which are very prone to pore blocking.

An improved catalyst performance and deactivation resistance is generally achieved for mesoporous zeolite catalysts. Considering the severe transport limitations inherent to the ZSM-22 zeolite catalyst, we suggest that a further investigation of the zeolites prepared and characterized here would be of significant interest. Pinpointing the causes of variations in catalytic performance and addressing single-parameter activity/deactivation dependencies is not straightforward, owing to the interdependence between the $\mathrm{Si} / \mathrm{Al}$ ratio, textural properties, particle size and shape, and acidity features demonstrated here. Certainly, having prepared and characterized a large number of catalyst samples is a prerequisite for tackling this challenging issue.

\section{Acknowledgments}


F.R and M.T.N thank to MINECO for financial support through projects MAT201571842-P and SEV-2012-0267. All the authors thank the Electron Microscopy Service of the Universitat Politècnica de València.

\section{References}

[1] S.M. Auerbach, K.A. Carrado, P.K. Dutta, Handbook of Zeolite Science and Technology, Marcel Dekker, Inc., 2003.

[2] A. Dyer, An introduction to zeolite molecular sieves, 1988.

[3] A. Corma, Inorganic Solid Acids and Their Use in Acid-Catalyzed Hydrocarbon Reactions, Chemical Reviews, 95 (1995) 559-614.

[4] A. Corma, From Microporous to Mesoporous Molecular Sieve Materials and Their Use in Catalysis, Chemical Reviews, 97 (1997) 2373-2420.

[5] B. Smit, T.L.M. Maesen, Towards a molecular understanding of shape selectivity, Nature, 451 (2008) 671-678.

[6] R. Chal, C. Gerardin, M. Bulut, D.S. van, Overview and industrial assessment of synthesis strategies towards zeolites with mesopores, ChemCatChem, 3 (2011) 67-81.

[7] M. Hartmann, Hierarchical zeolites: A proven strategy to combine shape selectivity with efficient mass transport, Angew. Chem., Int. Ed., 43 (2004) 5880-5882. 
[8] M.S. Holm, E. Taarning, K. Egeblad, C.H. Christensen, Catalysis with hierarchical zeolites, Catalysis Today, 168 (2011) 3-16.

[9] K. Li, J. Valla, J. Garcia-Martinez, Realizing the commercial potential of hierarchical zeolites: new opportunities in catalytic cracking, ChemCatChem, 6 (2014) 46-66.

[10] S. Mitchell, A.B. Pinar, J. Kenvin, P. Crivelli, J. Karger, J. Perez-Ramirez, Structural analysis of hierarchically organized zeolites, Nat. Commun., 6 (2015) 8633.

[11] K. Moller, T. Bein, Mesoporosity--a new dimension for zeolites, Chem Soc Rev, 42 (2013) 3689-3707.

[12] K. Na, M. Choi, R. Ryoo, Recent advances in the synthesis of hierarchically nanoporous zeolites, Microporous and Mesoporous Materials, 166 (2013) 3-19.

[13] K. Na, G. Somorjai, Hierarchically Nanoporous Zeolites and Their Heterogeneous Catalysis: Current Status and Future Perspectives, Catal Lett, 145 (2015) 193-213.

[14] J. Perez-Ramirez, C.H. Christensen, K. Egeblad, C.H. Christensen, J.C. Groen, Hierarchical zeolites: enhanced utilisation of microporous crystals in catalysis by advances in materials design, Chem. Soc. Rev., 37 (2008) 2530-2542.

[15] D.P. Serrano, J.M. Escola, P. Pizarro, Synthesis strategies in the search for hierarchical zeolites, Chem. Soc. Rev., 42 (2013) 4004-4035.

[16] J. Shi, Y. Wang, W. Yang, Y. Tang, Z. Xie, Recent advances of pore system construction in zeolite-catalyzed chemical industry processes, Chem. Soc. Rev., 44 (2015) 8877-8903.

[17] M.-C. Silaghi, C. Chizallet, P. Raybaud, Challenges on molecular aspects of dealumination and desilication of zeolites, Microporous Mesoporous Mater., 191 (2014) $82-96$.

[18] V. Valtchev, G. Majano, S. Mintova, J. Pérez-Ramírez, Tailored crystalline microporous materials by post-synthesis modification, Chemical Society Reviews, 42 (2013) 263-290.

[19] D. Verboekend, J. Perez-Ramirez, Design of hierarchical zeolite catalysts by desilication, Catal. Sci. Technol., 1 (2011) 879-890.

[20] S. Abello, J. Perez-Ramirez, Accelerated generation of intracrystalline mesoporosity in zeolites by microwave-mediated desilication, Phys. Chem. Chem. Phys., 11 (2009) 2959-2963.

[21] M. Bjoergen, F. Joensen, H.M. Spangsberg, U. Olsbye, K.-P. Lillerud, S. Svelle, Methanol to gasoline over zeolite H-ZSM-5: Improved catalyst performance by treatment with $\mathrm{NaOH}$, Appl. Catal., A, 345 (2008) 43-50.

[22] C.H. Christensen, K. Johannsen, E. Toernqvist, I. Schmidt, H. Topsoe, C.H. Christensen, Mesoporous zeolite single crystal catalysts: Diffusion and catalysis in hierarchical zeolites, Catal. Today, 128 (2007) 117-122.

[23] S. Gopalakrishnan, A. Zampieri, W. Schwieger, Mesoporous ZSM-5 zeolites via alkali treatment for the direct hydroxylation of benzene to phenol with N2O, J. Catal., 260 (2008) 193-197.

[24] J.C. Groen, J.C. Jansen, J.A. Moulijn, J. Perez-Ramirez, Optimal AluminumAssisted Mesoporosity Development in MFI Zeolites by Desilication, J. Phys. Chem. B, 108 (2004) 13062-13065.

[25] J.C. Groen, J.A. Moulijn, J. Perez-Ramirez, Decoupling mesoporosity formation and acidity modification in ZSM-5 zeolites by sequential desilication-dealumination, Microporous Mesoporous Mater., 87 (2005) 153-161.

[26] J.C. Groen, J.A. Moulijn, J. Perez-Ramirez, Desilication: on the controlled generation of mesoporosity in MFI zeolites, J. Mater. Chem., 16 (2006) 2121-2131. 
[27] J.C. Groen, L.A.A. Peffer, J.A. Moulijn, J. Perez-Ramirez, Mesoporosity development in ZSM-5 zeolite upon optimized desilication conditions in alkaline medium, Colloids Surf., A, 241 (2004) 53-58.

[28] J.C. Groen, L.A.A. Peffer, J.A. Moulijn, J. Perez-Ramirez, On the introduction of intracrystalline mesoporosity in zeolites upon desilication in alkaline medium, Microporous Mesoporous Mater., 69 (2004) 29-34.

[29] J.C. Groen, L.A.A. Peffer, J.A. Moulijn, J. Perez-Ramirez, Mechanism of hierarchical porosity development in MFI zeolites by desilication: The role of aluminum as a pore-directing agent, Chem. - Eur. J., 11 (2005) 4983-4994.

[30] J.C. Groen, W. Zhu, S. Brouwer, S.J. Huynink, F. Kapteijn, J.A. Moulijn, J. PerezRamirez, Direct demonstration of enhanced diffusion in mesoporous ZSM-5 zeolite obtained via controlled desilication, J Am Chem Soc, 129 (2007) 355-360.

[31] M.S. Holm, S. Svelle, F. Joensen, P. Beato, C.H. Christensen, S. Bordiga, M. Bjorgen, Assessing the acid properties of desilicated ZSM-5 by FTIR using CO and 2,4,6-trimethylpyridine (collidine) as molecular probes, Appl. Catal., A, 356 (2009) 2330 .

[32] F.C. Meunier, D. Verboekend, J.-P. Gilson, J.C. Groen, J. Perez-Ramirez, Influence of crystal size and probe molecule on diffusion in hierarchical ZSM-5 zeolites prepared by desilication, Microporous Mesoporous Mater., 148 (2012) 115-121.

[33] S. Mitchell, N.-L. Michels, K. Kunze, J. Perez-Ramirez, Visualization of hierarchically structured zeolite bodies from macro to nano length scales, Nat. Chem., 4 (2012) 825-831.

[34] M. Ogura, S.-y. Shinomiya, J. Tateno, Y. Nara, M. Nomura, E. Kikuchi, M. Matsukata, Alkali-treatment technique - new method for modification of structural and acid-catalytic properties of ZSM-5 zeolites, Appl. Catal., A, 219 (2001) 33-43.

[35] S. Svelle, L. Sommer, K. Barbera, P.N.R. Vennestrom, U. Olsbye, K.P. Lillerud, S. Bordiga, Y.-H. Pan, P. Beato, How defects and crystal morphology control the effects of desilication, Catal. Today, 168 (2011) 38-47.

[36] F. Thibault-Starzyk, I. Stan, S. Abello, A. Bonilla, K. Thomas, C. Fernandez, J.-P. Gilson, J. Perez-Ramirez, Quantification of enhanced acid site accessibility in hierarchical zeolites - The accessibility index, J. Catal., 264 (2009) 11-14.

[37] D. Verboekend, S. Mitchell, M. Milina, J.C. Groen, J. Perez-Ramirez, Full Compositional Flexibility in the Preparation of Mesoporous MFI Zeolites by Desilication, J. Phys. Chem. C, 115 (2011) 14193-14203.

[38] M. Ogura, E. Kikuchi, M. Matsukata, 11-P-30 - MFI zeolite with uniform mesopores created by alkali treatment, in: F.F.F.D.R. A. Galarneau, J. Vedrine (Eds.) Studies in Surface Science and Catalysis, Elsevier, 2001, pp. 216.

[39] F.L. Bleken, K. Barbera, F. Bonino, U. Olsbye, K.P. Lillerud, S. Bordiga, P. Beato, T.V.W. Janssens, S. Svelle, Catalyst deactivation by coke formation in microporous and desilicated zeolite H-ZSM-5 during the conversion of methanol to hydrocarbons, J. Catal., 307 (2013) 62-73.

[40] J. Perez-Ramirez, S. Abello, A. Bonilla, J.C. Groen, Tailored mesoporosity development in zeolite crystals by partial detemplation and desilication, Adv. Funct. Mater., 19 (2009) 164-172.

[41] W. Aslam, M.A.B. Siddiqui, B. Rabindran Jermy, A. Aitani, J. Cejka, S. AlKhattaf, Selective synthesis of linear alkylbenzene by alkylation of benzene with 1dodecene over desilicated zeolites, Catal. Today, 227 (2014) 187-197.

[42] K.P. de Jong, J. Zecevic, H. Friedrich, P.E. de Jongh, M. Bulut, S. van Donk, R. Kenmogne, A. Finiels, V. Hulea, F. Fajula, Zeolite Y Crystals with Trimodal Porosity 
as Ideal Hydrocracking Catalysts, Angew. Chem., Int. Ed., 49 (2010) 10074-10078, S10074/10071-S10074/10078.

[43] A. Bonilla, D. Baudouin, J. Perez-Ramirez, Desilication of ferrierite zeolite for porosity generation and improved effectiveness in polyethylene pyrolysis, J. Catal., 265 (2009) 170-180.

[44] M.H.M. Ahmed, O. Muraza, A.M. Al Amer, K. Miyake, N. Nishiyama, Development of hierarchical EU-1 zeolite by sequential alkaline and acid treatments for selective dimethyl ether to propylene (DTP), Appl. Catal., A, 497 (2015) 127-134.

[45] D. Verboekend, J.C. Groen, J. Perez-Ramirez, Interplay of Properties and Functions upon Introduction of Mesoporosity in ITQ-4 Zeolite, Adv. Funct. Mater., 20 (2010) 1441-1450.

[46] J.C. Groen, T. Sano, J.A. Moulijn, J. Perez-Ramirez, Alkaline-mediated mesoporous mordenite zeolites for acid-catalyzed conversions, J. Catal., 251 (2007) 2127.

[47] A.N.C. van laak, R.W. Gosselink, S.L. Sagala, J.D. Meeldijk, P.E. de Jongh, K.P. de Jong, Alkaline treatment on commercially available aluminum rich mordenite, Appl. Catal., A, 382 (2010) 65-72.

[48] B. Gil, L. Mokrzycki, B. Sulikowski, Z. Olejniczak, S. Walas, Desilication of ZSM-5 and ZSM-12 zeolites: Impact on textural, acidic and catalytic properties, Catal. Today, 152 (2010) 24-32.

[49] C. Baerlocher, L.B. McCusker, D.H. Olson, Atlas of zeolite framework types, Elsevier, 2007.

[50] G.T. Kokotailo, J.L. Schlenker, F.G. Dwyer, E.W. Valyocsik, The framework topology of ZSM-22: a high-silica zeolite, Zeolites, 5 (1985) 349-351.

[51] B. Marler, Silica-ZSM-22: synthesis and single crystal structure refinement, Zeolites, 7 (1987) 393-397.

[52] S.A.I. Barri, G.W. Smith, D. White, D. Young, Structure of Theta-1, the first unidimensional medium-pore high-silica zeolite, Nature (London), 312 (1984) 533-534.

[53] R.M. Highcock, G.W. Smith, D. Wood, Structure of the new zeolite theta-1 determined from x-ray powder data, Acta Crystallogr., Sect. C: Cryst. Struct. Commun., C41 (1985) 1391-1394.

[54] S. Liu, J. Ren, H. Zhang, E. Lv, Y. Yang, Y.-W. Li, Synthesis, characterization and isomerization performance of micro/mesoporous materials based on H-ZSM-22 zeolite, J. Catal., 335 (2016) 11-23.

[55] J.A. Martens, D. Verboekend, K. Thomas, G. Vanbutsele, J. Perez-Ramirez, J.-P. Gilson, Hydroisomerization and hydrocracking of linear and multibranched long model alkanes on hierarchical Pt/ZSM-22 zeolite, Catal. Today, Ahead of Print.

[56] P. Matias, C.C. Sa, I. Graca, J.M. Lopes, A.P. Carvalho, R.F. Ramoa, M. Guisnet, Desilication of a TON zeolite with $\mathrm{NaOH}$ : Influence on porosity, acidity and catalytic properties, Appl. Catal., A, 399 (2011) 100-109.

[57] C. Sá Couto, P. Matias, E.T. Santos, A. Fernandes, I. Graça, J.M. Lopes, M.F. Ribeiro, Towards a Deep Desilication/Dealumination of NU-10 Zeolite: ShapeSelectivity Regulation, European Journal of Inorganic Chemistry, 2012 (2012) 41904199.

[58] D. Verboekend, A.M. Chabaneix, K. Thomas, J.-P. Gilson, J. Perez-Ramirez, Mesoporous ZSM-22 zeolite obtained by desilication: peculiarities associated with crystal morphology and aluminum distribution, CrystEngComm, 13 (2011) 3408-3416.

[59] D. Verboekend, K. Thomas, M. Milina, S. Mitchell, J. Perez-Ramirez, J.-P. Gilson, Towards more efficient monodimensional zeolite catalysts: n-alkane hydroisomerisation on hierarchical ZSM-22, Catal. Sci. Technol., 1 (2011) 1331-1335. 
[60] P. del Campo, W.A. Slawinski, R. Henry, M.W. Erichsen, S. Svelle, P. Beato, D. Wragg, U. Olsbye, Time- and space-resolved high energy operando X-ray diffraction for monitoring the methanol to hydrocarbons reaction over H-ZSM-22 zeolite catalyst in different conditions, Surf. Sci., 648 (2016) 141-149.

[61] M. Dyballa, U. Obenaus, M. Rosenberger, A. Fischer, H. Jakob, E. Klemm, M. Hunger, Post-synthetic improvement of H-ZSM-22 zeolites for the methanol-to-olefin conversion, Microporous Mesoporous Mater., 233 (2016) 26-30.

[62] C. Martinez, E.J. Doskocil, A. Corma, Improved THETA-1 for light olefins oligomerization to diesel: influence of textural and acidic properties, Top. Catal., 57 (2014) 668-682.

[63] C. Fernandez, I. Stan, J.-P. Gilson, K. Thomas, A. Vicente, A. Bonilla, J. PerezRamirez, Hierarchical ZSM-5 Zeolites in Shape-Selective Xylene Isomerization: Role of Mesoporosity and Acid Site Speciation, Chem. - Eur. J., 16 (2010) 6224-6233, S6224/6221-S6224/6224.

[64] M. Milina, S. Mitchell, N.-L. Michels, J. Kenvin, J. Perez-Ramirez, Interdependence between porosity, acidity, and catalytic performance in hierarchical ZSM-5 zeolites prepared by post-synthetic modification, J. Catal., 308 (2013) 398-407.

[65] D. Tzoulaki, A. Jentys, J. Perez-Ramirez, K. Egeblad, J.A. Lercher, On the location, strength and accessibility of Bronsted acid sites in hierarchical ZSM-5 particles, Catal. Today, 198 (2012) 3-11.

[66] T. Biemelt, C. Selzer, F. Schmidt, G. Mondin, A. Seifert, K. Pinkert, S. Spange, T. Gemming, S. Kaskel, Hierarchical porous zeolite ZSM-58 derived by desilication and desilication re-assembly, Microporous Mesoporous Mater., 187 (2014) 114-124.

[67] F. Schmidt, M.R. Lohe, B. Buechner, F. Giordanino, F. Bonino, S. Kaskel, Improved catalytic performance of hierarchical ZSM-5 synthesized by desilication with surfactants, Microporous Mesoporous Mater., 165 (2013) 148-157.

[68] W.C. Yoo, X. Zhang, M. Tsapatsis, A. Stein, Synthesis of mesoporous ZSM-5 zeolites through desilication and re-assembly processes, Microporous Mesoporous Mater., 149 (2012) 147-157.

[69] I.I. Ivanova, I.A. Kasyanov, A.A. Maerle, V.I. Zaikovskii, Mechanistic study of zeolites recrystallization into micro-mesoporous materials, Microporous and Mesoporous Materials, 189 (2014) 163-172.

[70] I.I. Ivanova, A.S. Kuznetsov, E.E. Knyazeva, F. Fajula, F. Thibault-Starzyk, C. Fernandez, J.P. Gilson, Design of hierarchically structured catalysts by mordenites recrystallization: Application in naphthalene alkylation, Catalysis Today, 168 (2011) 133-139.

[71] Y.P. Khitev, Y.G. Kolyagin, I.I. Ivanova, O.A. Ponomareva, F. Thibault-Starzyk, J.P. Gilson, C. Fernandez, F. Fajula, Synthesis and catalytic properties of hierarchical micro/mesoporous materials based on FER zeolite, Microporous and Mesoporous Materials, 146 (2011) 201-207.

[72] S. Abello, A. Bonilla, J. Perez-Ramirez, Mesoporous ZSM-5 zeolite catalysts prepared by desilication with organic hydroxides and comparison with $\mathrm{NaOH}$ leaching, Appl. Catal., A, 364 (2009) 191-198.

[73] K. Mlekodaj, K. Tarach, J. Datka, K. Gora-Marek, W. Makowski, Porosity and accessibility of acid sites in desilicated ZSM-5 zeolites studied using adsorption of probe molecules, Microporous Mesoporous Mater., 183 (2014) 54-61.

[74] J. Perez-Ramirez, D. Verboekend, A. Bonilla, S. Abello, Zeolite Catalysts with Tunable Hierarchy Factor by Pore-Growth Moderators, Adv. Funct. Mater., 19 (2009) 3972-3979. 
[75] K. Sadowska, K. Gora-Marek, M. Drozdek, P. Kustrowski, J. Datka, J. Martinez Triguero, F. Rey, Desilication of highly siliceous zeolite ZSM-5 with $\mathrm{NaOH}$ and $\mathrm{NaOH} /$ tetrabutylamine hydroxide, Microporous Mesoporous Mater., 168 (2013) 195205.

[76] K. Sadowska, A. Wach, Z. Olejniczak, P. Kustrowski, J. Datka, Hierarchic zeolites: Zeolite ZSM-5 desilicated with $\mathrm{NaOH}$ and $\mathrm{NaOH} /$ tetrabutylamine hydroxide, Microporous Mesoporous Mater., 167 (2013) 82-88.

[77] K. Tarach, K. Gora-Marek, J. Tekla, K. Brylewska, J. Datka, K. Mlekodaj, W. Makowski, M.C. Igualada Lopez, J. Martinez Triguero, F. Rey, Catalytic cracking performance of alkaline-treated zeolite Beta in the terms of acid sites properties and their accessibility, J. Catal., 312 (2014) 46-57.

[78] D. Verboekend, M. Milina, S. Mitchell, J. Perez-Ramirez, Hierarchical Zeolites by Desilication: Occurrence and Catalytic Impact of Recrystallization and Restructuring, Cryst. Growth Des., 13 (2013) 5025-5035.

[79] D. Verboekend, J. Perez-Ramirez, Desilication Mechanism Revisited: Highly Mesoporous All-Silica Zeolites Enabled Through Pore-Directing Agents, Chem. - Eur. J., 17 (2011) 1137-1147, S1137/1131-S1137/1133.

[80] S. Teketel, U. Olsbye, K.-P. Lillerud, P. Beato, S. Svelle, Selectivity control through fundamental mechanistic insight in the conversion of methanol to hydrocarbons over zeolites, Microporous Mesoporous Mater., 136 (2010) 33-41.

[81] S. Teketel, W. Skistad, S. Benard, U. Olsbye, K.P. Lillerud, P. Beato, S. Svelle, Shape Selectivity in the Conversion of Methanol to Hydrocarbons: The Catalytic Performance of One-Dimensional 10-Ring Zeolites: ZSM-22, ZSM-23, ZSM-48, and EU-1, ACS Catal., 2 (2012) 26-37.

[82] S. Teketel, S. Svelle, K.-P. Lillerud, U. Olsbye, Shape-selective conversion of methanol to hydrocarbons over 10-ring unidirectional-channel acidic H-ZSM-22, ChemCatChem, 1 (2009) 78-81.

[83] D. Masih, T. Kobayashi, T. Baba, Hydrothermal synthesis of pure ZSM-22 under mild conditions, Chem. Commun. (Cambridge, U. K.), (2007) 3303-3305.

[84] K. Hayasaka, D. Liang, W. Huybrechts, B.R. De Waele, K.J. Houthoofd, P. Eloy, E.M. Gaigneaux, G. van Tendeloo, J.W. Thybaut, G.B. Marin, J.F.M. Denayer, G.V. Baron, P.A. Jacobs, C.E.A. Kirschhock, J.A. Martens, Formation of ZSM-22 zeolite catalytic particles by fusion of elementary nanorods, Chem. - Eur. J., 13 (2007) 1007010077.

[85] S.J.S.K.S.W. Gregg, Adsorption, surface area, and porosity, Academic Press, London; New York, 1982.

[86] J.C. Groen, L.A.A. Peffer, J. Perez-Ramirez, Pore size determination in modified micro- and mesoporous materials. Pitfalls and limitations in gas adsorption data analysis, Microporous Mesoporous Mater., 60 (2003) 1-17.

[87] J.C. Groen, J. Perez-Ramirez, Critical appraisal of mesopore characterization by adsorption analysis, Appl. Catal., A, 268 (2004) 121-125.

[88] G. Leofanti, M. Padovan, G. Tozzola, B. Venturelli, Surface area and pore texture of catalysts, Catal. Today, 41 (1998) 207-219.

[89] R. Borade, A. Adnot, S. Kaliaguine, Acid sites in Al-ZSM-22 and Fe-ZSM-22, Zeolites, 11 (1991) 710-719.

[90] S. Bordiga, C. Lamberti, F. Bonino, A. Travert, F. Thibault-Starzyk, Probing zeolites by vibrational spectroscopies, Chem. Soc. Rev., 44 (2015) 7262-7341.

[91] F. Geobaldo, S. Fiorilli, B. Onida, G. Giordano, A. Katovic, E. Garrone, An FTIR Study of Zeolite Theta-1, J. Phys. Chem. B, 107 (2003) 1258-1262. 
[92] K. Barbera, F. Bonino, S. Bordiga, T.V.W. Janssens, P. Beato, Structuredeactivation relationship for ZSM-5 catalysts governed by framework defects, J. Catal., 280 (2011) 196-205.

[93] W. Lutz, C.H. Ruscher, D. Heidemann, Determination of the framework and nonframework [SiO2] and [AlO2] species of steamed and leached faujasite type zeolites: calibration of IR, NMR, and XRD data by chemical methods, Microporous Mesoporous Mater., 55 (2002) 193-202.

[94] R.D. Shannon, K.H. Gardner, R.H. Staley, G. Bergeret, P. Gallezot, A. Auroux, The nature of the nonframework aluminum species formed during the dehydroxylation of H-Y, J. Phys. Chem., 89 (1985) 4778-4788.

[95] S. Bordiga, I. Roggero, P. Ugliengo, A. Zecchina, V. Bolis, G. Artioli, R. Buzzoni, G. Marra, F. Rivetti, G. Spanò, Characterisation of defective silicalites, Journal of the Chemical Society, Dalton Transactions, (2000) 3921-3929.

[96] S. Bordiga, P. Ugliengo, A. Damin, C. Lamberti, G. Spoto, A. Zecchina, G. Spano, R. Buzzoni, L. Dalloro, F. Rivetti, Hydroxyls nests in defective silicalites and strained structures derived upon dehydroxylation: vibrational properties and theoretical modelling, Topics in Catalysis, 15 (2001) 43-52.

[97] R.F. Lobo, Introduction to the structural chemistry of zeolites, in, Marcel Dekker, Inc., 2003, pp. 65-89.

[98] R. Dessau, E. Valyocsik, N. Goeke, Aluminum zoning in ZSM-5 as revealed by selective silica removal, Zeolites, 12 (1992) 776-779.

[99] C. Emeis, Determination of integrated molar extinction coefficients for infrared absorption bands of pyridine adsorbed on solid acid catalysts, Journal of Catalysis, 141 (1993) 347-354. 


\section{Table 1}

Summary of the desilication conditions applied in this work. $-a t$, $-a t s$ and -tba corresponds to alkaline, alkaline-CTAB and alkaline-TBAOH treated samples, respectively.

\begin{tabular}{|c|c|c|c|c|c|c|}
\hline \multirow{2}{*}{ Sample code } & \multicolumn{3}{|c|}{ Desilication treatment (M) } & \multirow{2}{*}{$\begin{array}{c}\mathrm{T} \\
\left({ }^{\circ} \mathrm{C}\right)\end{array}$} & \multirow{2}{*}{$\begin{array}{l}\mathrm{t} \\
\text { (h) }\end{array}$} & \multirow{2}{*}{$\begin{array}{l}\mathrm{S} / \mathrm{L}^{\mathrm{a}} \\
\left(\mathrm{g} \mathrm{l}^{-1}\right)\end{array}$} \\
\hline & $\mathrm{C}_{\mathrm{NaOH}}$ & $\mathrm{C}_{\mathrm{CTAB}}$ & $\mathrm{C}_{\mathrm{TBAOH}}$ & & & \\
\hline $\begin{array}{l}c \text {-ZSM-22-at1 } \\
m \text {-ZSM-22-at1 }\end{array}$ & 0.2 & - & - & \multirow{2}{*}{80} & \multirow{2}{*}{2} & \multirow{2}{*}{33} \\
\hline $\begin{array}{l}c \text {-ZSM-22-at2 } \\
m \text {-ZSM-22-at2 }\end{array}$ & 0.5 & - & - & & & \\
\hline $\begin{array}{l}c \text {-ZSM-22-ats } 1 \\
m \text {-ZSM-22-ats } 1\end{array}$ & 0.25 & 0.05 & - & \multirow{2}{*}{80} & \multirow{2}{*}{24} & \multirow{2}{*}{50} \\
\hline $\begin{array}{l}c \text {-ZSM-22-ats2 } \\
m \text {-ZSM-22-ats2 }\end{array}$ & 0.5 & 0.05 & - & & & \\
\hline $\begin{array}{l}c \text {-ZSM-22-tbal } \\
m \text {-ZSM-22-tbal } \\
c \text {-ZSM-22-tba2 } \\
m \text {-ZSM-22-tba2 }\end{array}$ & 0.12 & - & 0.08 & 80 & 0.5 & 30 \\
\hline
\end{tabular}

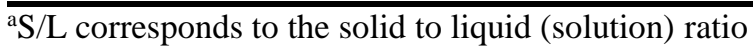


Table 2

Composition, morphology and textural properties for the ZSM-22 studied samples.

\begin{tabular}{|c|c|c|c|c|c|c|c|c|}
\hline \multirow{2}{*}{ Sample } & \multirow{2}{*}{$\begin{array}{l}\text { Yield }^{\mathrm{a}} \\
(\%)\end{array}$} & \multicolumn{2}{|c|}{$\mathrm{Si} / \mathrm{Al}$ ratio $\left(\mathrm{mol} \mathrm{mol}^{-1}\right)$} & \multicolumn{3}{|c|}{ Pore volume $\left(\mathrm{cm}^{3} \mathrm{~g}^{-1}\right)$} & \multicolumn{2}{|c|}{ Surface area $\left(\mathrm{m}^{2} \mathrm{~g}^{-1}\right)$} \\
\hline & & MP-AES & Predicted $^{b}$ & $V_{\text {total }}{ }^{\mathrm{c}}$ & $V_{\text {micro }}{ }^{\mathrm{d}}$ & $V_{\text {ext }}^{\mathrm{e}}$ & $\overline{S_{B E T}}$ & $S_{\text {ext }}^{\mathrm{d}}$ \\
\hline$c$-ZSM-22 & - & 49 & - & 0.15 & 0.08 & 0.07 & 232 & 33 \\
\hline$c$-ZSM-22-at1 & 60 & 35 & 29 & 0.30 & 0.08 & 0.22 & 257 & 65 \\
\hline$c$-ZSM-22-at2 & 46 & 31 & 23 & 0.53 & 0.08 & 0.45 & 247 & 60 \\
\hline c-ZSM-22-atl-HCl & - & 48 & - & 0.56 & 0.08 & 0.48 & 276 & 82 \\
\hline$c-\mathrm{ZSM}-22-a t 2-\mathrm{HCl}$ & - & 46 & - & 0.53 & 0.07 & 0.46 & 251 & 68 \\
\hline$c$-ZSM-22-ats 1 & 82 & 40 & 40 & 0.26 & 0.07 & 0.19 & 233 & 61 \\
\hline$c$-ZSM-22-ats2 & 51 & 29 & 25 & 0.48 & 0.06 & 0.42 & 263 & 118 \\
\hline c-ZSM-22-ats $1-H C l$ & - & 50 & - & 0.29 & 0.08 & 0.21 & 257 & 58 \\
\hline c-ZSM-22-ats2- $\mathrm{HCl}$ & - & 48 & - & 0.52 & 0.07 & 0.45 & 296 & 114 \\
\hline$c$-ZSM-22-tbal & 88 & 33 & 43 & 0.23 & 0.08 & 0.14 & 232 & 43 \\
\hline$c-Z S M-22-t b a 2$ & 75 & 32 & 37 & 0.21 & 0.05 & 0.16 & 171 & 35 \\
\hline c-ZSM-22-tbal-HCl & - & 54 & - & 0.26 & 0.09 & 0.17 & 257 & 49 \\
\hline$c-Z S M-22-t b a 2-H C l$ & - & 47 & - & 0.29 & 0.09 & 0.20 & 272 & 44 \\
\hline$c-Z S M-22-H C l$ & - & 52 & - & 0.14 & 0.08 & 0.06 & 214 & 21 \\
\hline$m$-ZSM-22 & - & 38 & - & 0.39 & 0.05 & 0.34 & 163 & 26 \\
\hline$m$-ZSM-22-at1 & 60 & 29 & 23 & 0.57 & 0.07 & 0.50 & 216 & 40 \\
\hline$m$-ZSM-22-at2 & 48 & 25 & 18 & 0.53 & 0.07 & 0.47 & 202 & 38 \\
\hline$m$-ZSM-22-at1-HCl & - & 36 & - & 0.56 & 0.08 & 0.48 & 236 & 39 \\
\hline$m-Z S M-22-a t 2-H C l$ & - & 37 & - & 0.59 & 0.08 & 0.51 & 257 & 45 \\
\hline$m$-ZSM-22-ats1 & 83 & 30 & 32 & 0.44 & 0.02 & 0.42 & 88 & 35 \\
\hline$m$-ZSM-22-ats2 & 58 & 22 & 22 & 0.34 & 0.01 & 0.33 & 47 & 23 \\
\hline$m$-ZSM-22-ats1- $\mathrm{HCl}$ & - & 34 & - & 0.23 & 0.03 & 0.20 & 108 & 29 \\
\hline$m$-ZSM-22-ats2- $\mathrm{HCl}$ & - & 21 & - & 0.14 & 0.01 & 0.13 & 47 & 21 \\
\hline$m$-ZSM-22-tbal & 90 & 28 & 34 & 0.39 & 0.06 & 0.33 & 178 & 22 \\
\hline$m-Z S M-22-t b a 2$ & 82 & 26 & 31 & 0.17 & 0.01 & 0.16 & 73 & 43 \\
\hline$m$-ZSM-22-tbal- $\mathrm{HCl}$ & - & 39 & - & 0.42 & 0.08 & 0.34 & 213 & 21 \\
\hline$m$-ZSM-22-tba2- $\mathrm{HCl}$ & - & 39 & - & 0.31 & 0.07 & 0.24 & 211 & 32 \\
\hline
\end{tabular}

${ }^{\mathrm{a}} 100$ - weight loss (\%) after desilication.

${ }^{\mathrm{b}}$ Calculated multiplying the $\mathrm{Si} / \mathrm{Al}$ ratio of the parent sample by the yield of the treatment.

${ }^{\mathrm{c}}$ Volume adsorbed at $p / p_{0}=0.99$.

${ }^{\mathrm{d}} t$-plot method. $V_{\text {ext }}$ and $S_{\text {ext }}$ represent the pore volume and surface area for all the pores except the micropores.

${ }^{\text {e }} V_{\text {total }}-V_{\text {micro }}$. 


\section{Table 3}

Acidic properties for selected ZSM-22 protonated zeolites.

\begin{tabular}{|c|c|c|c|c|c|}
\hline Sample & 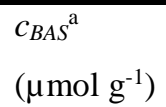 & 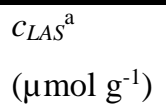 & $\begin{array}{l}c_{B A S}+c_{L A S} \\
\left(\mu \mathrm{mol} \mathrm{g}{ }^{-1}\right)\end{array}$ & $\begin{array}{l}c_{A l}^{\mathrm{b}} \\
\left.(\mu \mathrm{mol} \mathrm{g})^{-1}\right)\end{array}$ & $\begin{array}{l}\text { Acidic sites }^{\mathrm{c}} \\
(\%)\end{array}$ \\
\hline$c$-ZSM-22 & 240 & 30 & 270 & 340 & 79 \\
\hline$c$-ZSM-22-at1 & 240 & 130 & 370 & 476 & 78 \\
\hline$c$-ZSM-22-atl- $\mathrm{HCl}$ & - & - & - & 347 & - \\
\hline$c$-ZSM-22-ats 1 & 210 & 130 & 340 & 417 & 82 \\
\hline$c$-ZSM-22-ats $1-H C l$ & 220 & 100 & 320 & 333 & 96 \\
\hline$c$-ZSM-22-tba2 & 330 & 120 & 450 & 521 & 86 \\
\hline$c-\mathrm{ZSM}-22-t b a 2-\mathrm{HCl}$ & 280 & 70 & 350 & 355 & 98 \\
\hline$m$-ZSM-22 & 240 & 30 & 270 & 439 & 62 \\
\hline$m$-ZSM-22-at1 & 440 & 130 & 570 & 575 & 99 \\
\hline$m-Z S M-22-a t 1-H C l$ & 380 & 50 & 430 & 463 & 93 \\
\hline$m$-ZSM-22-ats1 & 150 & 40 & 190 & 556 & 34 \\
\hline$m$-ZSM-22-ats $1-H C l$ & 210 & 50 & 260 & 490 & 53 \\
\hline$m-Z S M-22-t b a l$ & 460 & 70 & 530 & 595 & 89 \\
\hline$m-Z S M-22-t b a l-H C l$ & 370 & 40 & 410 & 427 & 96 \\
\hline
\end{tabular}

a Amount of Brønsted or Lewis acid sites determined by IR spectroscopy of adsorbed pyridine.

bTotal Al concentration calculated from elemental analysis $=1 /(60 \times \mathrm{Si} / \mathrm{Al})$.

${ }^{\mathrm{c}}$ Total acid sites reached by pyridine adsorption $\left(c_{B A S}+c_{L A S}\right) /$ total $\mathrm{Al}$ concentration $\left(c_{A l}\right)$ in $\%$. 

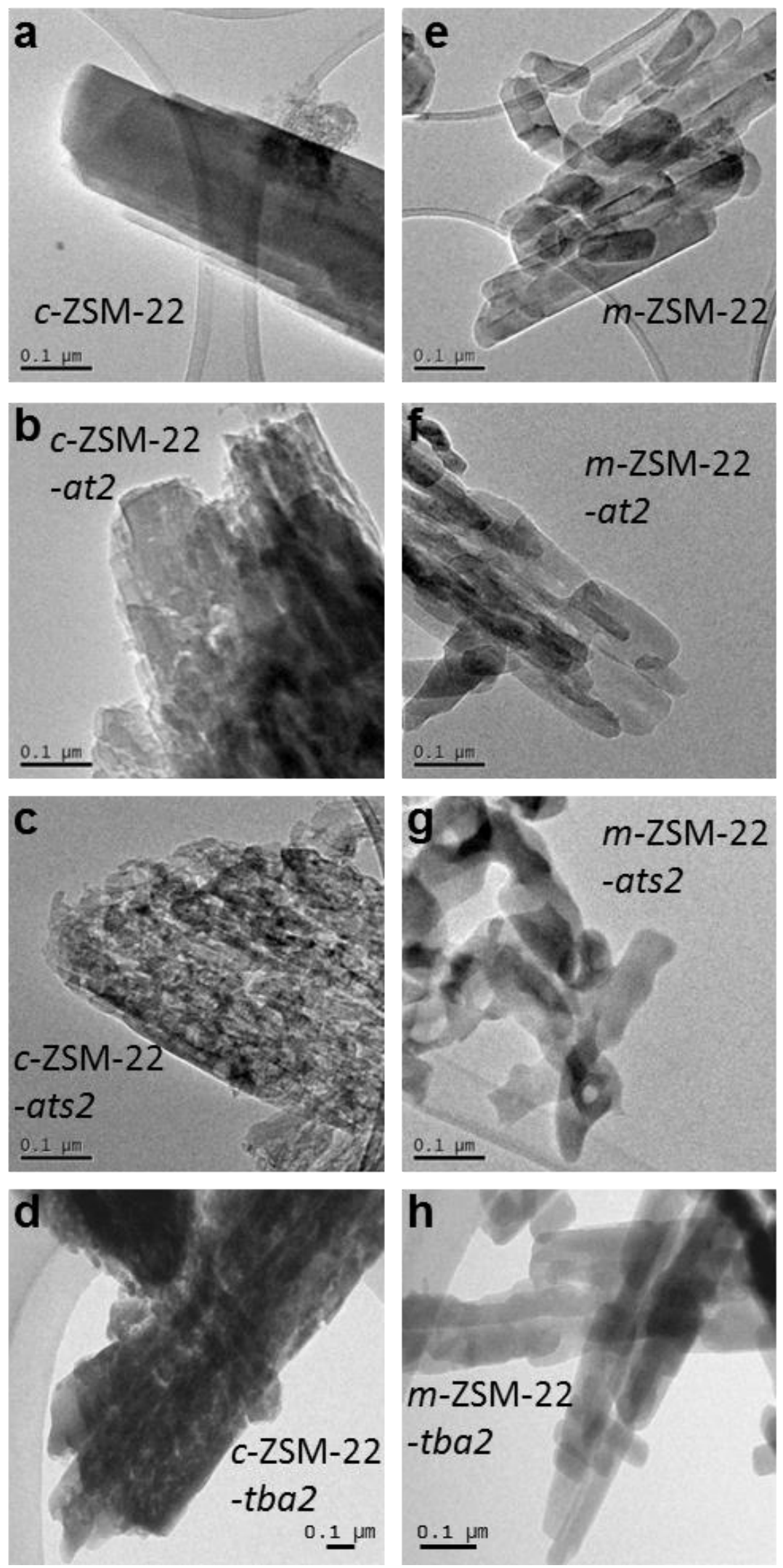

Fig. 1. TEM images for the parent and desilicated commercial and in house prepared ZSM-22 samples. 

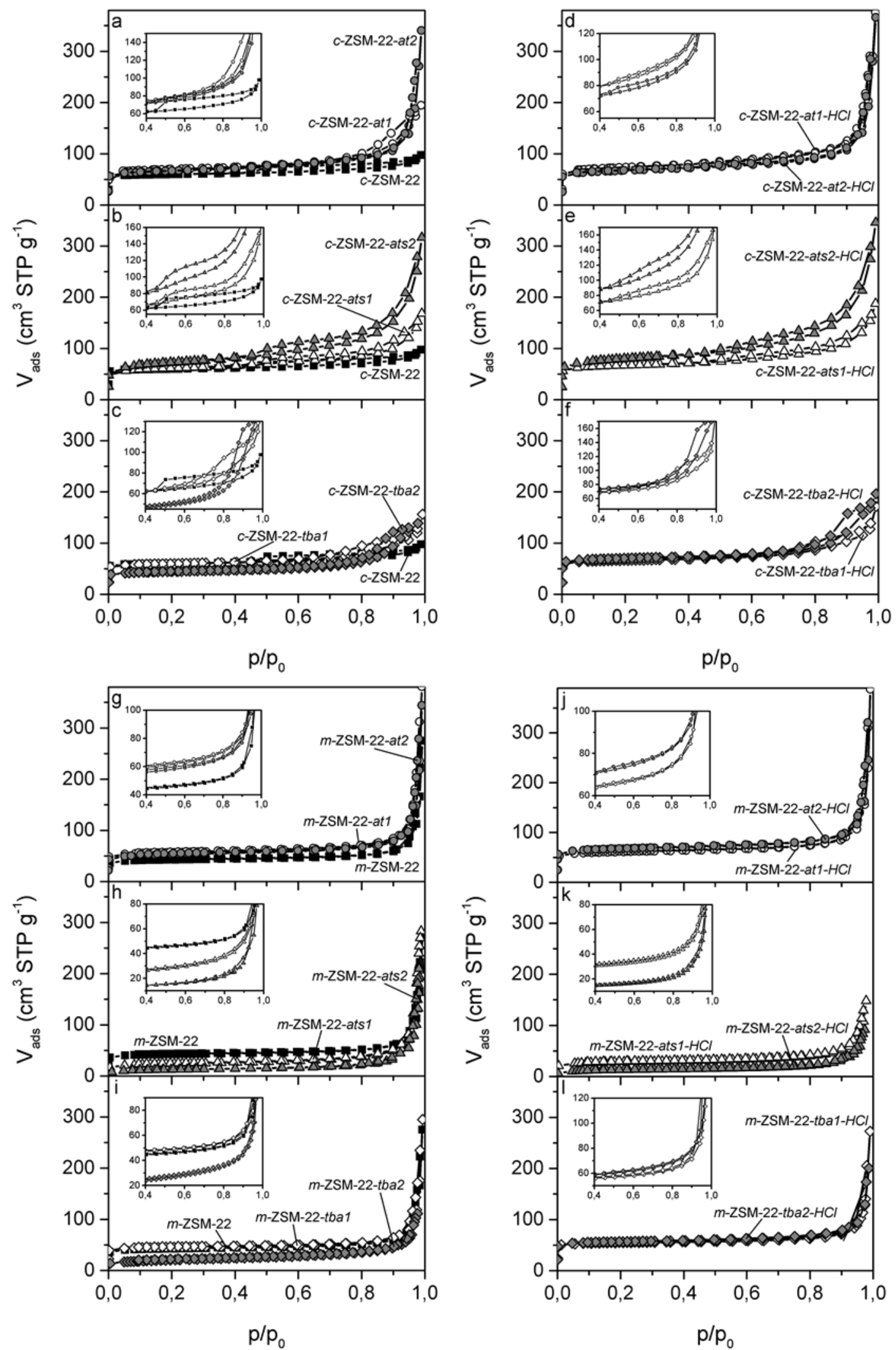

Fig. 2. Nitrogen adsorption-desorption isotherms for the parent, desilicated and acid washed ZSM-22 samples (a-f for commercial set, $\mathbf{g}-\mathbf{l}$ for the in house prepared set). The insets highlight particular regions of each plot. 

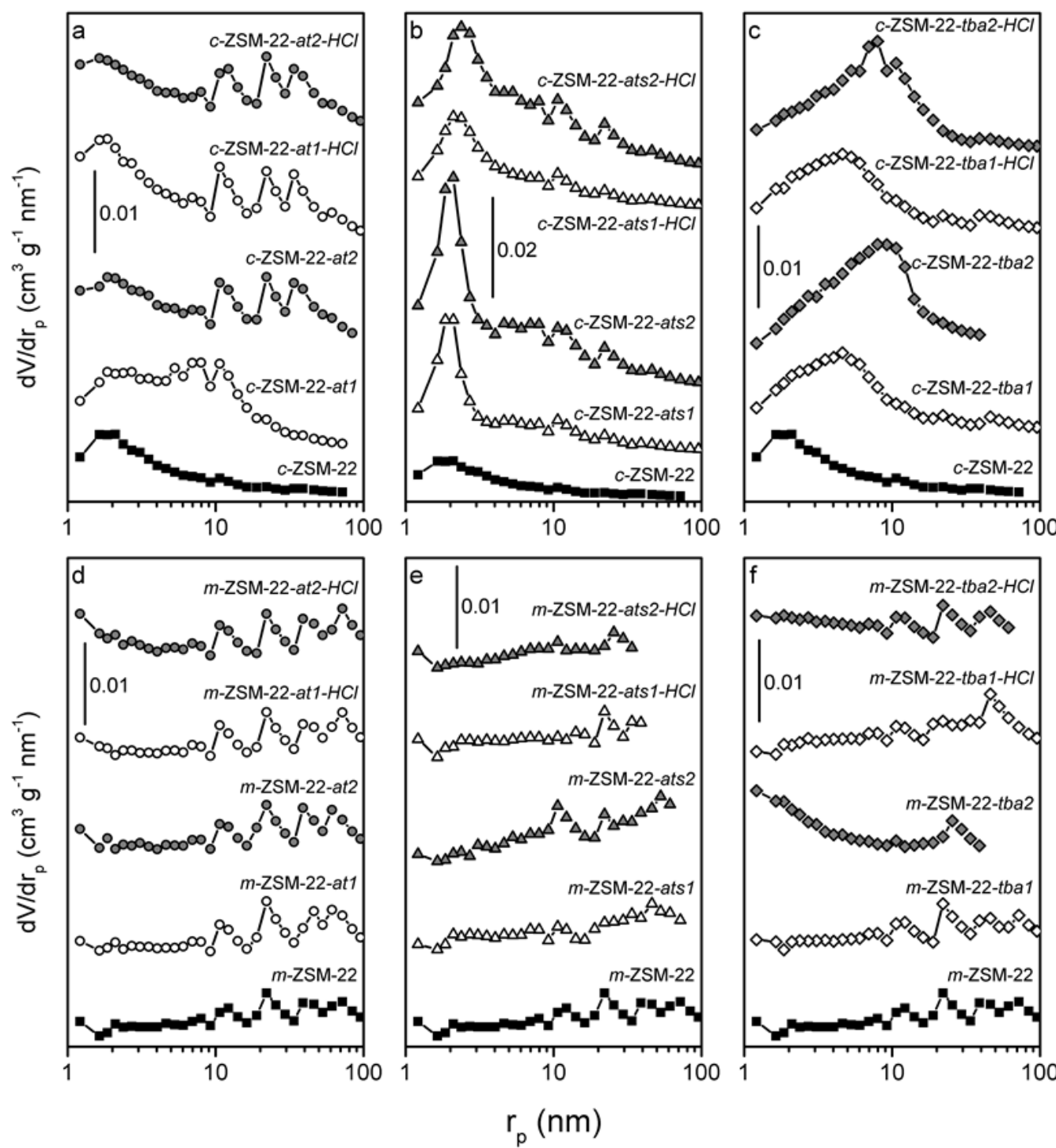

Fig. 3. Effect of the different desilication and acid washing on the BJH mesopore size distribution, as calculated from the adsorption branch of the isotherm, for the commercial (a-c) and in house prepared (df) ZSM-22 samples. 

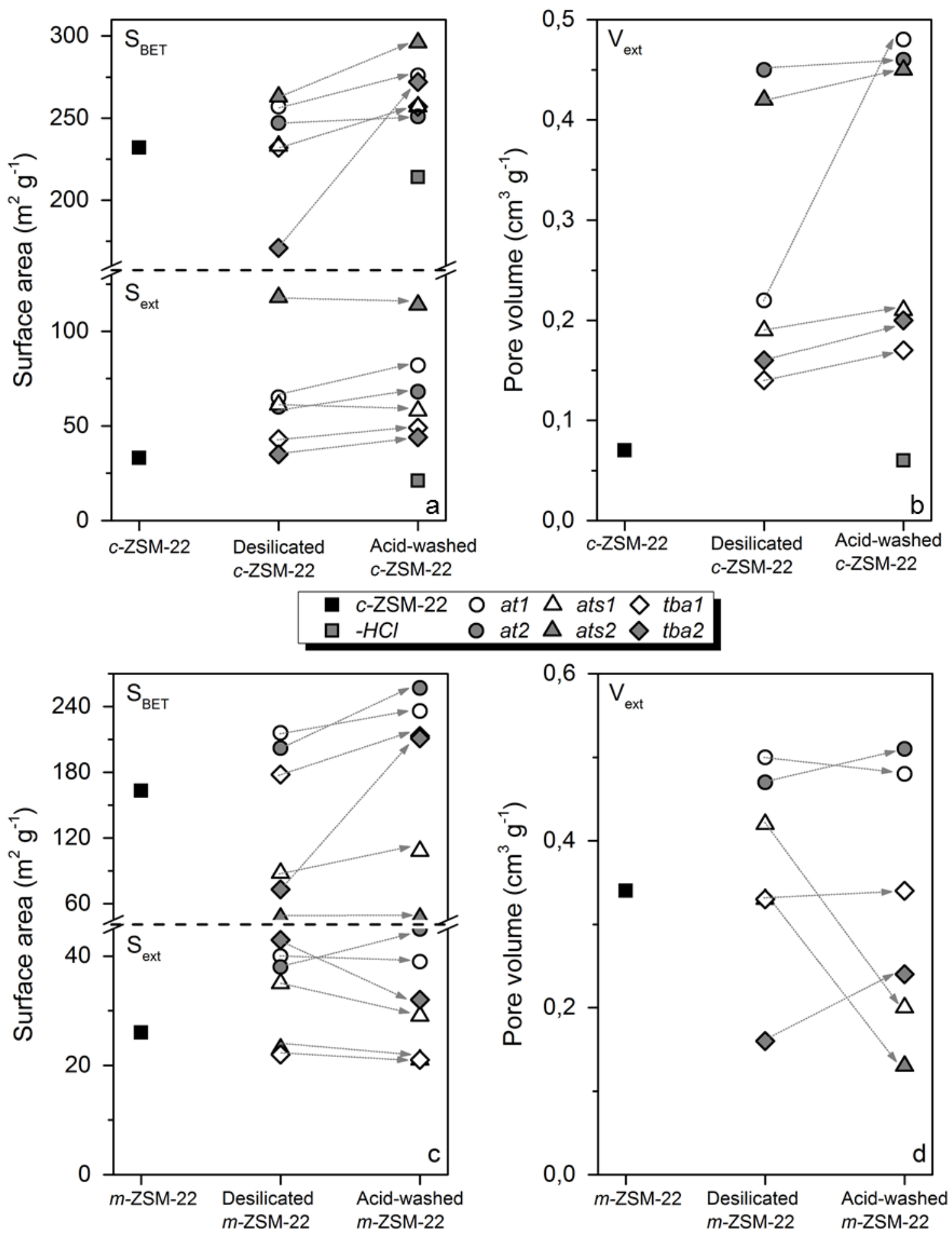

Fig. 4. Influence of the desilication and acid washing on the total and external surface area (a and c) and external pore volume (b and d) for the commercial (top panels) and in house prepared (bottom panels) ZSM-22 zeolites. 


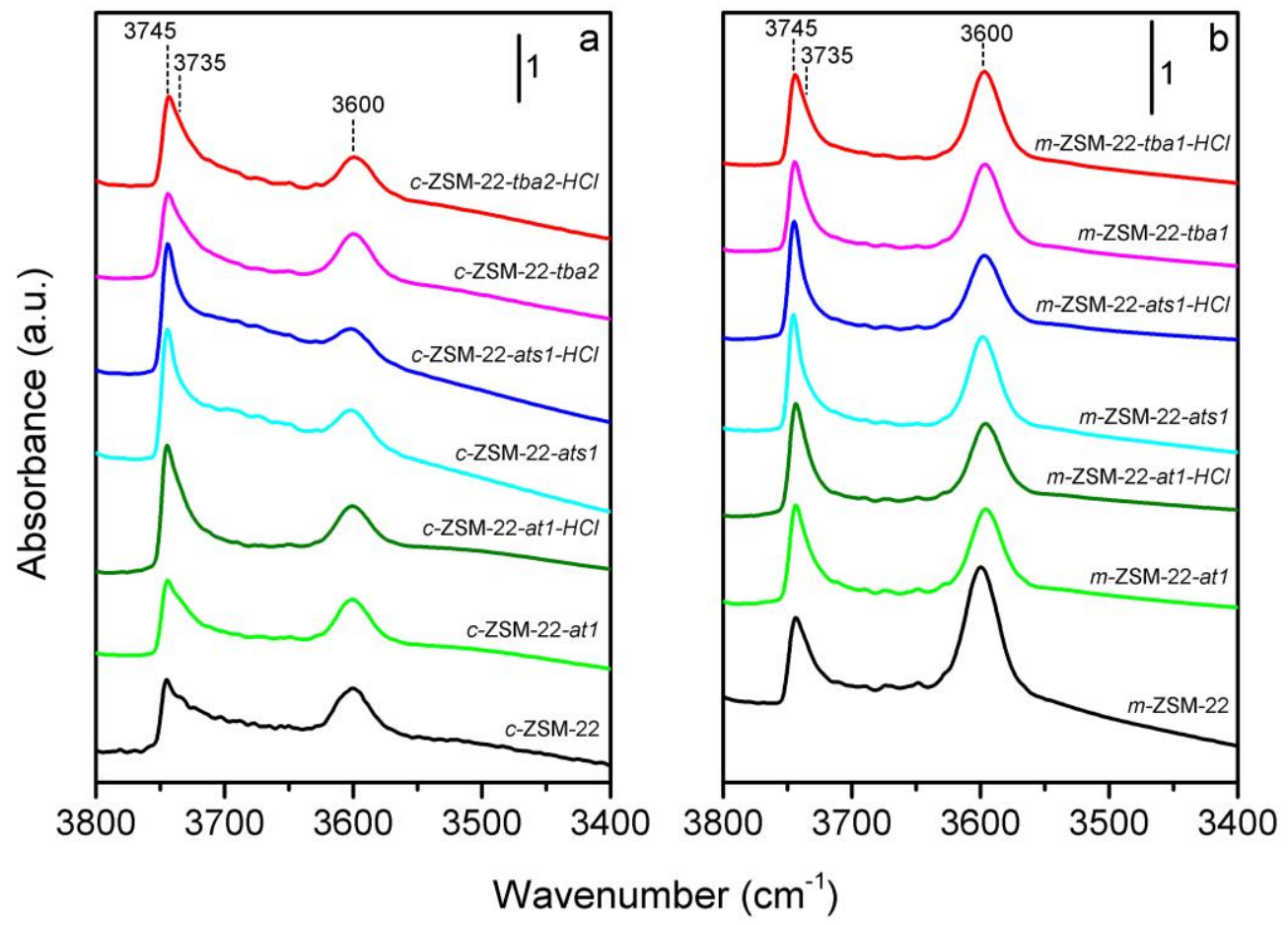

Fig. 5. FTIR spectra of the parent, desilicated and acid treated commercial (a) and in house prepared (b) ZSM-22 zeolites activated at $450{ }^{\circ} \mathrm{C}$ in the hydroxyl stretching region. 


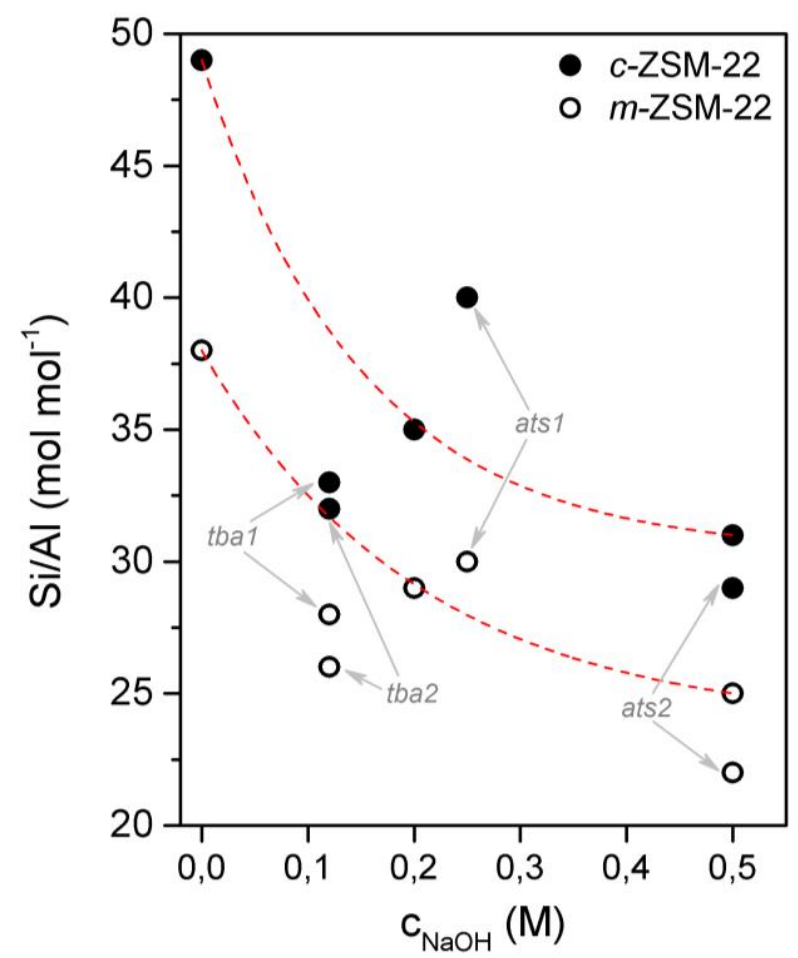

Fig. 6. $\mathrm{Si} / \mathrm{Al}$ ratio as a function of $\mathrm{NaOH}$ molar concentration for the commercial and in house prepared desilicated samples (the explanatory codes are listed in Table 1). The dashed line shows the reference effect of the only alkaline treatment. 

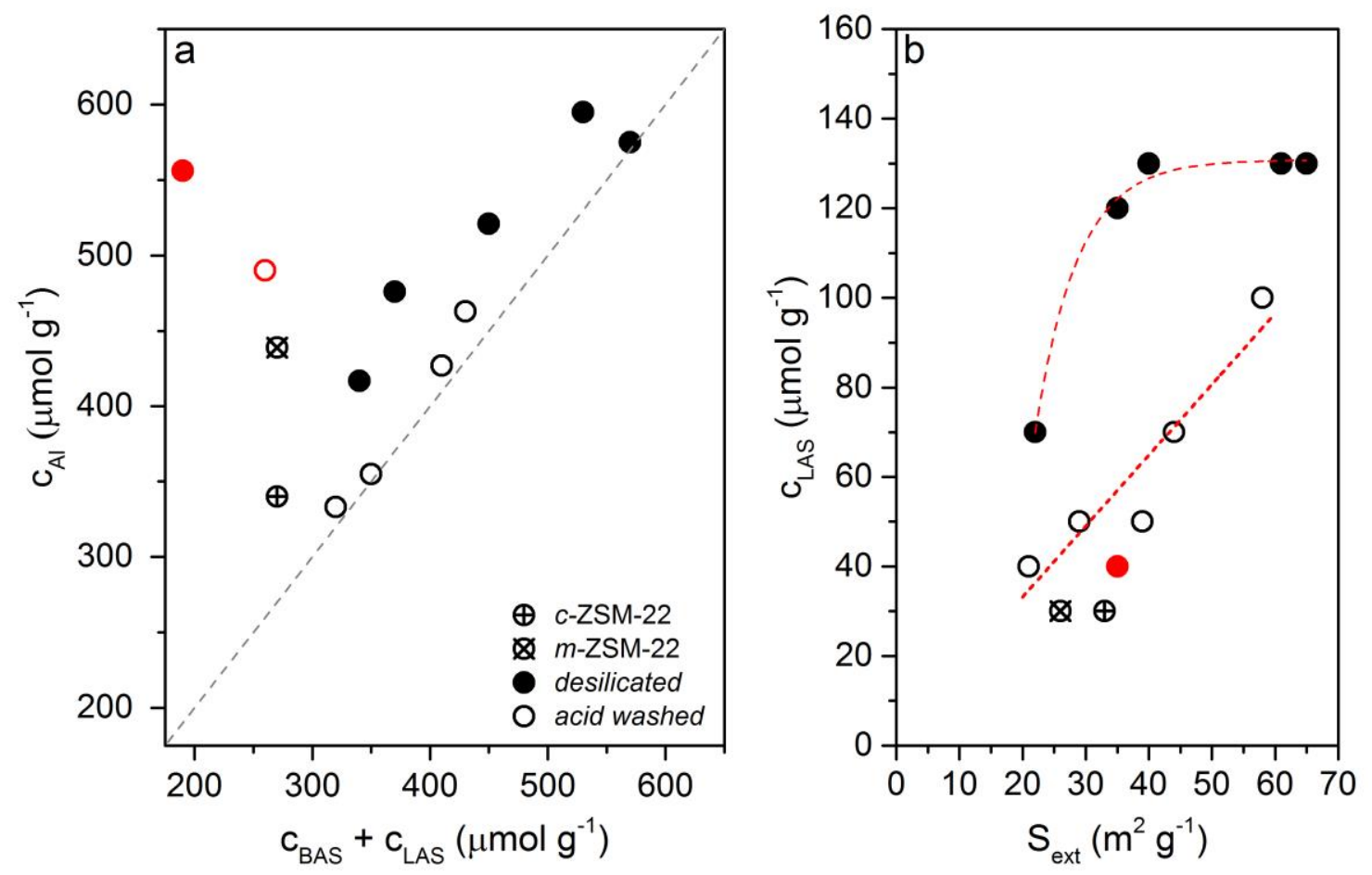

Fig. 7. (a) Correlation between total Al concentration and concentration of acidic sites (BAS + LAS) and (b) interdependence between Lewis acidity and external surface area for the parent and selected treated zeolites. 